\title{
Theory of Near-Adiabatic Collisions. III. Coupled Equations Arising from Expansions Involving Single-Center States
}

John B. Delos

William \& Mary, jbdelos@wm.edu

Follow this and additional works at: https://scholarworks.wm.edu/aspubs

Part of the Physics Commons

\section{Recommended Citation}

Delos, John B., Theory of Near-Adiabatic Collisions. III. Coupled Equations Arising from Expansions Involving Single-Center States (1981). Physical Review A, 23(5), 2301-2318.

https://doi.org/10.1103/PhysRevA.23.2301

This Article is brought to you for free and open access by the Arts and Sciences at W\&M ScholarWorks. It has been accepted for inclusion in Arts \& Sciences Articles by an authorized administrator of W\&M ScholarWorks. For more information, please contact scholarworks@wm.edu. 


\title{
Theory of near-adiabatic collisions. III. Coupled equations arising from expansions involving single-center states
}

\author{
J. B. Delos* \\ FOM-Institute for Atomic and Molecular Physics, Kruislaan 407, Amsterdam, The Netherlands
}

(Received 2 June 1980)

\begin{abstract}
The conventional quantum-mechanical formulation of near-adiabatic collision theory is known to have a number of defects. These defects arise because the usual description does not account for the displacement of electronic states with moving nuclei, or for the change of momentum of the electron as it jumps from one moving nucleus to the other. The purpose of this series of papers is to develop an improved theory, in which such effects are taken into account. In this paper, we show that displacement and momentum-transfer effects can be incorporated into the theory in a very simple way, provided that the wave function is expanded in terms of electronic states that have single-center character. (Linear combinations of single-center states are also permitted.) A particular form of such an expansion is proposed, and it is shown that this expansion leads to equations in which fictitious displacement couplings are eliminated and momentum-transfer terms are included. The work of this paper and of others on this subject leads to revised notions about the definition and meaning of nonadiabatic couplings.
\end{abstract}

\section{INTRODUCTION}

The quantum theory of slow atomic collisions has in the past started with an expansion of the full wave function $\Psi(\vec{R}, \vec{r})$ in terms of molecular states $\phi_{k}(\overrightarrow{\mathrm{r}}, \overrightarrow{\mathrm{R}})$ :

$$
\Psi(\overrightarrow{\mathrm{R}}, \overrightarrow{\mathrm{r}})=\sum_{k} \chi_{k}(\overrightarrow{\mathrm{R}}) \phi_{k}(\overrightarrow{\mathrm{r}} ; \overrightarrow{\mathrm{R}}) .
$$

Typically the basis functions $\phi_{k}(\overrightarrow{\mathrm{r}} ; \overrightarrow{\mathrm{R}})$ are taken to be eigenfunctions of the electronic part of the Hamiltonian, but other choices are possible, and frequently are more convenient. Assuming only that the basis functions are orthogonal, the expansion (1), inserted into the Schrödinger equation, leads to a set of coupled equations, which can be written in the form ${ }^{1}$

$$
\left\{(2 \mu)^{-1}\left[(-i \hbar \nabla)^{2}+2 \underline{\overrightarrow{\mathrm{P}}} \cdot(-i \hbar \vec{\nabla})+\underline{B}\right]+\underline{h}-E\right\} \underline{\chi}=0,
$$

where

$$
\begin{aligned}
& \overrightarrow{\mathrm{P}}_{j k}=\int \phi_{j}^{*}\left(-i \hbar \vec{\nabla}_{R} \phi_{k}\right)_{\mathrm{r}} d \overrightarrow{\mathrm{r}}, \\
& B_{j k}=\int \phi_{j}^{*}\left[\left(-i \hbar \nabla_{R}\right)^{2} \phi_{k}\right]_{\mathrm{r}} d \overrightarrow{\mathrm{r}},
\end{aligned}
$$

and all other notation is defined in Tables I, II, and III. These equations (2), determining the nuclear wave functions $\chi_{n}(\vec{R})$, constitute the original quantum-mechanical form of the perturbed-stationary-states (PSS) theory. ${ }^{2}$

It has been known for many years ${ }^{2-6}$ that these equations have a number of defects which render them inappropriate for the description of some collision processes: For example, Eqs. (2) contain infinite-range radial couplings, long-range $\left(\sim R^{-1}\right)$ angular couplings, "origin-dependent" terms, and nonphysical matrix elements for ion- ization. ${ }^{7}$ In addition, since the matrix elements do not contain momentum-transfer factors, Eqs. (2) do not go over to the correct classical limit, as formulated, for example, in Ref. 4.

The purpose of this series of papers is to develop a "corrected" quantum theory of slow collisions, one in which the above-mentioned defects do not appear. It was known that the strange couplings that appear in Eqs. (2) are all "fictitious": They represent only the displacement of electronic orbitals with the moving nuclei, and they do not correspond to real nonadiabatic effects that can lead to observed electronic transitions. In Ref. 3(b), it was shown that the displacement terms could be eliminated by making use of an ansatz [Eq. (5.17) of Ref. 3(b)] which was based upon a curvilinear heavy-particle coordinate. Although the resulting description is satisfactory for most purposes, it retains one undesirable limitation: The same curvilinear coordinate was used for all basis states.

As a result, the modified coupling matrix elements given in Ref. 3 still do not contain momentum-transfer factors, so they also do not go exactly to the correct classical limit. In addition, the use of the same coordinate for all states provides an unnecessary restriction on variational procedures. ${ }^{7,8}$

In this paper and elsewhere, ${ }^{9}$ we generalize the development given in Ref. 3(b), and eliminate this one remaining restriction. The central idea is an intersecting-wave picture, which requires the use of different coordinates for different basis states. Such an approach leads to coupled integrodifferential equations ${ }^{10}$; we will show that approximations appropriate for slow atomic collisions reduce those equations to a tractable 
form. We will arrive at a set of coupled second-order differential equations quite similar to (2), but with momentum-transfer effects included and fictitious displacement terms eliminated.

In its most general form, our development permits the use of any type of basis function, and different curvilinear coordinates for different basis states. In this very general framework, ${ }^{9}$ although the final results are simple, the derivation of those results is rather long. For this reason, we present here a much easier derivation. However, this simpler derivation is only possible if we are willing to impose a different restriction: The basis functions must be either single-center states or arbitrary linear combinations of single-center states. Single-center states are states that are clearly attached to a particular center in the molecule: one or the other of the nuclei, the geometric center, the center of mass of the nuclei, or some other identifiable point. (In Ref. 4 we referred to these as class $V$ or class $F$ states..$\left.^{11}\right)$

The precise nature of this restriction will become clear later. Here let us only emphasize that the present development permits the use of $a r b i$ trary linear combinations of single-center states, including combinations involving states from different centers. Such basis sets are used in many calculations.

The methods presented here are applicable to multielectron systems, but to keep the equations simple, we develop them explicitly only for oneelectron (or "effectively one-electron") systems. The generalization to multielectron systems is considered briefly at the end.

(Since this paper is an extension of previous work, the reader may find it helpful first to look at Sec. III A of Ref. 3(b). The classical analog of the ideas presented here is given in Sec. II and III of Ref. 4. Other parts of those references are not essential for understanding the present paper.)

\section{DEFINITIONS: PROPERTIES OF BASIS STATES}

Definitions of coordinates are the same as those given in Ref. 3(b). After the motion of the center of mass of the system has been separated, the relative positions of electron and nuclei are conveniently specified by any one of three pairs of Jacobi coordinates. These are shown in Fig. 1, and their relationships are given in Table I. As in Ref. 3 (but unlike Ref。4), the components $(x, y, z)$ of the vector $\overrightarrow{\mathrm{r}}$ are defined relative to a "spacefixed" (i.e., nonrotating) frame of reference. Associated with each coordinate is a reduced mass, given in Table II. Later, we will make use of mass-scaled coordinates, defined by ${ }^{3(b), 12}$
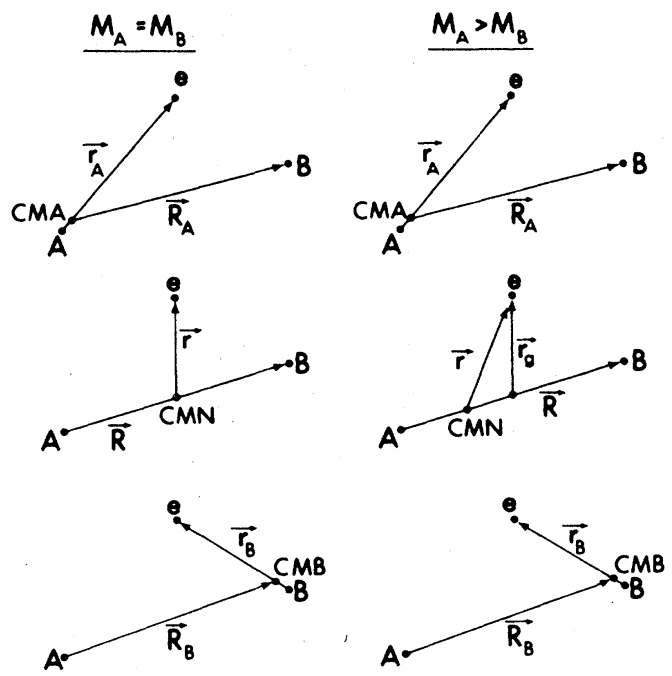

FIG. 1. Three Jacobi coordinate systems for relative coordinates of two heavy particles $A, B$, and an electron $e$, for equal masses, $M_{A}=M_{B}$, and for unequal masses, $M_{A}>M_{B}$. CMN denotes center of mass of $A, B$.

$$
C_{J}^{m}=M_{\text {assoc }}^{1 / 2} C_{J}
$$

where $M_{\text {assoc }}$ represents the associated mass and $C_{J}$ is the coordinate.

The full Hamiltonian for the electron and nuclei is

$$
H=T+V,
$$

with

$$
\begin{aligned}
T & =-\frac{\hbar^{2}}{2 \mu_{A}} \nabla_{R_{A}}^{2}-\frac{\hbar^{2}}{2 m_{a}} \nabla_{r_{A}}^{2} \\
& =-\frac{\hbar^{2}}{2 \mu_{B}} \nabla_{R_{B}}^{2}-\frac{\hbar^{2}}{2 m_{B}} \nabla_{r_{B}}^{2}
\end{aligned}
$$

TABLE I. Relations of coordinates.

$$
\begin{aligned}
& \lambda=\left(M_{A}-M_{B}\right)\left(M_{A}+M_{B}\right) \\
& \frac{1}{2}(1+\lambda)=M_{A} /\left(M_{A}+M_{B}\right), \frac{1}{2}(1-\lambda)=M_{B} /\left(M_{A}+M_{B}\right) \\
& \overrightarrow{\mathrm{r}}=\overrightarrow{\mathrm{r}}_{g}+\frac{1}{2} \lambda \overrightarrow{\mathrm{R}} \\
& \overrightarrow{\mathrm{r}}_{A}=\overrightarrow{\mathrm{r}}_{\mathrm{g}}+\frac{1}{2} \overrightarrow{\mathrm{R}}=\overrightarrow{\mathrm{r}}+\frac{1}{2}(1-\lambda) \overrightarrow{\mathrm{R}} \\
& \overrightarrow{\mathrm{r}}_{B}=\overrightarrow{\mathrm{r}}_{g}-\frac{1}{2} \overrightarrow{\mathrm{R}}=\overrightarrow{\mathrm{r}}-\frac{1}{2}(1+\lambda) \overrightarrow{\mathrm{R}} \\
& \overrightarrow{\mathrm{R}}_{A}=\frac{M_{A}+\frac{1}{2} m_{0}}{M_{A}+m_{0}} \overrightarrow{\mathrm{R}}-\frac{m_{0}}{M_{A}+m_{0}} \overrightarrow{\mathrm{r}}_{g} \\
& \quad=\frac{M_{A}+\frac{1}{2}(1+\lambda) m_{0}}{M_{A}+m_{0}} \overrightarrow{\mathrm{R}}-\frac{m_{0}}{M_{A}+m_{0}} \overrightarrow{\mathrm{r}} \\
& \overrightarrow{\mathrm{R}}_{B}=\frac{M_{B}+\frac{1}{2} m_{0}}{M_{B}+m_{0}} \overrightarrow{\mathrm{R}}+\frac{m_{0}}{M_{B}+m_{0}} \overrightarrow{\mathrm{r}}_{g} \\
& \quad=\frac{M_{B}+\frac{1}{2}(1-\lambda) m_{0}}{M_{B}+m_{0}} \overrightarrow{\mathrm{R}}+\frac{m_{0}}{M_{B}+m_{0}} \overrightarrow{\mathrm{r}}
\end{aligned}
$$


TABLE II. Reduced masses corresponding to coordinates.

\begin{tabular}{lll}
\hline Coordinate & & Reduced mass \\
$\overrightarrow{\mathbf{r}}^{0}$ & $m_{0}$ & Rest mass of electron \\
$\overrightarrow{\mathrm{R}}_{A}^{0}$ & $M_{A}$ & Rest mass of $A$ nucleus \\
$\overrightarrow{\mathrm{R}}_{B}^{0}$ & $M_{B}$ & Rest mass of $B$ nucleus \\
$\overrightarrow{\mathrm{R}}_{\mathrm{c} . \mathrm{m} .}^{0}$ & $M_{T}=M_{A}+M_{B}+m_{0}$ & Total mass of system \\
$\overrightarrow{\mathrm{r}}_{A}$ & $m_{A}=m_{0} M_{A} /\left(m_{0}+M_{A}\right)$ & Electron reduced mass, channel $A$ \\
$\overrightarrow{\mathrm{R}}_{A}$ & $\mu_{A}=\left(m_{0}+M_{A}\right) M_{B} / M_{T}$ & Nuclear reduced mass, channel $A$ \\
$\overrightarrow{\mathrm{r}}_{B}$ & $m_{B}=m_{0} M_{B} /\left(m_{0}+M_{B}\right)$ & Electron reduced mass, channel $B$ \\
$\overrightarrow{\mathrm{R}}_{B}$ & $\mu_{B}=\left(m_{0}+M_{B}\right) M_{A} / M_{T}$ & Nuclear reduced mass, channel $B$ \\
$\overrightarrow{\mathbf{r}}$ & $m=m_{0}\left(M_{A}+M_{B}\right) / M_{T}$ & Molecular electron reduced mass \\
$\overrightarrow{\mathrm{R}}$ & $\mu=M_{A} M_{B} /\left(M_{A}+M_{B}\right)$ & Molecular nuclear reduced mass \\
\hline \hline
\end{tabular}

$$
=-\frac{\hbar^{2}}{2 \mu} \nabla_{R}^{2}-\frac{\hbar^{2}}{2 m} \nabla_{r}^{2} .
$$

$V$ is the potential energy of interaction of electron and nuclei, which might have the form

$$
V=+\hat{Z}_{A} \hat{Z}_{B} / R-\hat{Z}_{A} / r_{A}-\hat{Z}_{B} / r_{B} \text {. }
$$

[For a system containing one active electron outside of a closed "inert" shell, $V$ is the effective potential, which will be more complicated than (5d)].

We will make use of an expansion of the wave function in electronic basis states. The elementary basis states are assumed to be square integrable, and it is assumed that at least some of them correlate asymptotically to the important initial and final atomic eigenstates. Furthermore, each of the elementary basis states is assumed to have single-center character-it must be associated with and propagating with a particular center in the molecule.

The concept of "single-center character" is understood by quantum chemists. For example, any basis function that depends only on $\vec{r}_{A}$ (or on $\overrightarrow{\mathrm{r}}_{B}$ ) is rigorously a single-center $A$ (or $B$ ) state. The simplest examples are the $1 s_{A}$ and $1 s_{B}$ states of the separated atoms,

$$
\begin{aligned}
& \phi_{1 s_{A}}=N_{A} \exp \left(-\xi_{A} r_{A}\right), \\
& \phi_{1 s_{B}}=N_{B} \exp \left(-\xi_{B} r_{B}\right) .
\end{aligned}
$$

Certain other types of states are also permitted in this theory. The states may have parameters, like orbital exponents, that smoothly vary to optimize some property of the basis. It is essential to the present formulation, however, that any varying parameters must be regarded as functions of the conjugate Jacobi heavy-particle coordinate. Thus we also permit, for example, the basis func- tion

$$
\phi_{1 s_{A}^{\prime}}\left(\overrightarrow{\mathbf{r}}_{A} ; \overrightarrow{\mathrm{R}}_{A}\right)=N_{A}\left(\overrightarrow{\mathrm{R}}_{A}\right) \exp \left[-\xi\left(\overrightarrow{\mathrm{R}}_{A}\right)\right] r_{A},
$$

where $\xi\left(\vec{R}_{A}\right)$ varies smoothly as a function of $\vec{R}_{A}$. Another type of parametric variation arises if rotating basis functions are used: the vector $\vec{R}_{A}$ specifies a direction in space, and the angular factor in a basis function could be oriented ("quantized") relative to this axis..$^{13}$ Such rotating basis functions are much more appropriate than spacefixed functions for describing molecular stationary states or slow atomic collisions.

Three parenthetical remarks may be made about these basis states.

(1) As long as no parametric variation is allowed, single-center states can be given a rigorous mathematical definition: they are functions only of any one electronic coordinate. However, if parametric variation is permitted, a rigorous definition is not so simple. Any function [including $\left.\phi_{1 s_{B}}\left(\overrightarrow{\mathrm{r}}_{B}\right)\right]$ can be written as a function of $\overrightarrow{\mathrm{r}}_{A}$ and $\overrightarrow{\mathrm{R}}_{A}$, but this does not mean that it qualifies as an $A$-centered state. In actual calculations, the elementary basis functions are usually defined in such a way that their single-center character is apparent. If, however, one cannot be sure that a given basis state is associated with a particular center, then, to the same degree, one cannot be sure that the theory developed here will be useful for a collision calculation involving that basis state.

(2) In molecular structure calculations, the variable parameters are usually regarded as functions of $\vec{R}$, not of $\vec{R}_{A}$ or $\vec{R}_{B}$. Given such a function defined on a surface of constant $\vec{R}$ in the configuration space, one must map it or displace it onto a surface of constant $\vec{R}_{A}$ or $\vec{R}_{B}$. This can be done by replacing $\vec{R}$ by $\vec{R}_{A}$ or $\vec{R}_{B}$ in the analytic 
formula for the function. [See Ref. 3(b) or Ref. 9 for a more detailed discussion.]

(3) The basis states need not be centered on the nuclei. If they are functions of some other electronic coordinate $\overrightarrow{\mathrm{r}}_{\alpha}$ then the conjugate Jacobi heavy-particle coordinate $\vec{R}_{\alpha}$ may be defined such that the kinetic energy contains no cross terms of the form $\vec{\nabla}_{R_{\alpha}} \cdot \vec{\nabla}_{r_{\alpha}}$. Equivalently, the pair of coordinates $\overrightarrow{\mathrm{r}}_{\alpha}, \overrightarrow{\mathrm{R}}_{\alpha}$ may be defined by a rotation in mass-scaled coordinate space. ${ }^{3(b), 12}$

\section{EXPANSION OF THE WAVE FUNCTION AND COUPLED INTEGRO-DIFFERENTIAL EQUATIONS}

Having specified the properties of the basis functions that are permitted in the present formulation, we now expand $\Psi$ in terms of these functions, and obtain a set of coupled integro-differential equations. The basic hypothesis is that $\Psi$ can be expressed to sufficient accuracy by the ansatz

$$
\Psi=\sum_{k=1}^{M} \chi_{k}\left(\overrightarrow{\mathrm{R}}_{k}\right) \phi_{k}\left(\overrightarrow{\mathrm{r}}_{k} ; \overrightarrow{\mathrm{R}}_{k}\right) \text {. }
$$

In this expansion, the basis functions $\phi_{k}$ are chosen, and the "coefficients" $\chi_{k}$ are to be determined. Each basis function is expressed in terms of the pair of Jacobi coordinates $\left(\overrightarrow{\mathbf{r}}_{k}, \overrightarrow{\mathrm{R}}_{k}\right)$ appropriate to the center with which $\phi_{k}$ is associated; then $\chi_{k}$ is assumed to be a function of the corresponding heavy-particle coordinate $\overrightarrow{\mathrm{R}}_{k}$. In most cases, only basis states centered on the nuclei are used, and $\left(\overrightarrow{\mathrm{r}}_{k}, \overrightarrow{\mathrm{R}}_{k}\right)$ means either $\left(\overrightarrow{\mathrm{r}}_{A}, \overrightarrow{\mathrm{R}}_{A}\right)$ or $\left(\overrightarrow{\mathrm{r}}_{B}, \overrightarrow{\mathrm{R}}_{B}\right)$.

Let us examine the meaning of this ansatz. Although Eqs. (1) and (6) are superficially similar, the former could represent an expansion in a complete set of orthogonal functions (spanning the function space associated with the electronic coordinate $\vec{r}$ ), while the latter represents an expansion in a finite set of nonorthogonal functions which are not members of any particular complete set. More important, various terms in the expansion (6) are given in terms of different coordinates. Although any term, or the whole sum, could be written in terms of any one pair of Jacobi coordinates, the separation into a product $\chi_{k}\left(\overrightarrow{\mathrm{R}}_{k}\right)$ $\phi_{k}\left(\overrightarrow{\mathrm{r}}_{k} ; \overrightarrow{\mathrm{R}}_{k}\right)$, with $\phi_{k}$ a one-center state, holds only in the original coordinates given for each term.

A special case of this ansatz (6) would be $\mathrm{i}^{10}$

$$
\Psi=\chi_{1}\left(\overrightarrow{\mathrm{R}}_{A}\right) \phi_{1 s_{A}}\left(\overrightarrow{\mathrm{r}}_{A}\right)+\chi_{2}\left(\overrightarrow{\mathrm{R}}_{B}\right) \phi_{1 s_{B}}\left(\overrightarrow{\mathrm{r}}_{B}\right) 。
$$

To make its meaning more clear, consider the function

$$
\exp \left(i k Z_{A}\right) \exp \left(-\left|z_{A}\right|\right)+\exp \left(i k Z_{B}\right) \exp \left(-\left|z_{B}\right|\right) .
$$

If this function were reexpressed in mass-scaled coordinates, it would represent the crossing of two wave trains (Fig. 2). Each of these wave trains is oscillatory and propagating in a particular direction (toward increasing $Z_{A}^{m}$ or $Z_{B}^{m}$ ), but each is a localized exponential wave in the perpendicular direction $\left(z_{A}^{m}\right.$ or $\left.z_{B}^{m}\right)$. In contrast, a corresponding simplification of (1) would give

$$
\exp (i k Z)\left[\exp \left(-\left|z_{A}\right|\right)+\exp \left(-\left|z_{B}\right|\right)\right]
$$

which is quite different from (8a): For example, the nodal lines of the real part of each term in (8b) would all be vertical in Fig. 2. To see that (8a) is the better description, we only need to note that it satisfies the scattering boundary conditions, while $(8 b)$ does not. The essential postulate of the present approach is that it is possible to obtain a sufficiently accurate representation of $\Psi$ in the molecular interaction region, as well as in the channels, by a reasonable number of terms in the ansatz (6).

From this ansatz, it is a simple matter to obtain coupled integro-differential equations for the functions $\chi_{k}\left(\overrightarrow{\mathrm{R}}_{k}\right)_{0}{ }^{10}$ From Eqs. (4) and (5), the Hamiltonian is written as a function of any pair of Jacobi coordinates as

$$
\begin{aligned}
& H=H^{(k)}\left(\overrightarrow{\mathrm{r}}_{k}, \overrightarrow{\mathrm{R}}_{k}\right)=-\frac{\hbar^{2}}{2 \mu_{k}} \nabla_{R_{k}}^{2}+h_{k}, \\
& h_{k}=-\frac{\hbar^{2}}{2 m_{k}} \nabla_{r_{k}}^{2}+V,
\end{aligned}
$$

and we write

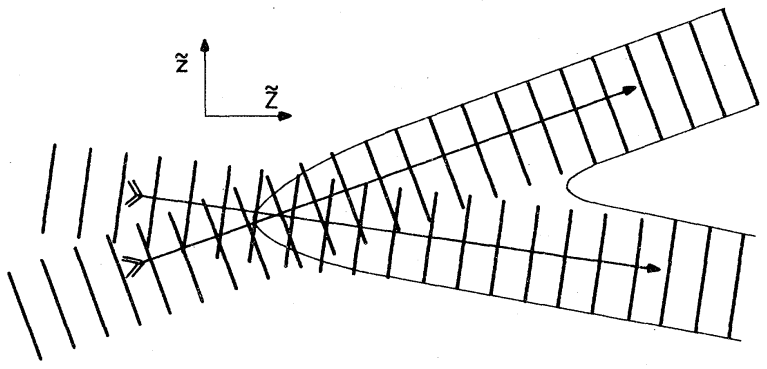

FIG. 2. Intersecting wave trains corresponding to Eq. (8a). The waves propagate in the direction of the arrows, but they are localized in the directions perpendicular to their propagation. The heavy lines represent nodes of the real part of each term in (8a). The light lines are equipotentials of $V=-\left|z_{A}\right|^{-1}-\left|z_{B}\right|^{-1}+|z|^{-1}$ drawn as functions of mass-scaled coordinates $z^{m}=\tilde{z}$, $Z^{m}=\tilde{Z}$ for $M_{A}=18, M_{B}=6, m_{0}=1$. These equipotentials are the same as those shown in Fig. 6 of Ref. 3(b). One sees in this figure that the wave trains are appropriately aligned with the channels. In contrast, nodes of individual terms of Eq. (8b) would be vertical lines in this figure, and they would not be properly aligned with the geometry of the potential. [Plane-wave trains (8) would extend indefinitely to the left, but ansatz (7) would give waves only near classically allowed regions.] 


$$
\begin{aligned}
\mathcal{O} & =(H-E) \Psi=\sum_{k}\left[H^{(k)}\left(\overrightarrow{\mathrm{r}}_{k}, \overrightarrow{\mathrm{R}}_{k}\right)-E\right] \chi_{\chi_{k}}\left(\overrightarrow{\mathrm{R}}_{k}\right) \phi_{k}\left(\overrightarrow{\mathrm{r}}_{k} ; \overrightarrow{\mathrm{R}}_{k}\right) \\
& =\sum_{k}\left(-\frac{\hbar^{2}}{2 \mu_{k}}\left(\phi_{k} \nabla_{R_{k}}^{2} \chi_{k}+2 \vec{\nabla}_{R_{k}} \phi_{k} \cdot \vec{\nabla}_{R_{k}} \chi_{k}+\chi_{k} \nabla_{R_{k}}^{2} \phi_{k}\right)+\chi_{k} h_{k} \phi_{k}-E \chi_{k} \phi_{k}\right) .
\end{aligned}
$$

If the ansatz (6) is a good one, then 0 will be everywhere small. This function, which is originally a sum of terms, with various terms expressed in different coordinates, can be reexpressed as a function of any one pair of Jacobi coordinates $\left(\overrightarrow{\mathrm{r}}_{j}, \overrightarrow{\mathrm{R}}_{j}\right)$. For each $j(j=1 \cdots M)$, we reexpress $\mathcal{O}$ in this way,

$$
\Theta=\theta_{f}\left(\vec{r}_{j} ; \vec{R}_{j}\right) \text {, }
$$

multiply by $\phi_{j}\left(\overrightarrow{\mathrm{r}}_{j} ; \overrightarrow{\mathrm{R}}_{j}\right)$, integrate over electronic coordinates $\overrightarrow{\mathrm{r}}_{j}$ holding $\overrightarrow{\mathrm{R}}_{j}$ fixed, and set the result to zero:

$$
\int_{\text {P1хed } \overrightarrow{\mathrm{R}}_{j}} \phi *\left(\overrightarrow{\mathrm{r}}_{j} ; \overrightarrow{\mathrm{R}}_{j}\right) \Theta_{j}\left(\overrightarrow{\mathrm{r}}_{j}, \overrightarrow{\mathrm{R}}_{j}\right) d \overrightarrow{\mathrm{r}}_{j}=0 .
$$

Equations (12) are a set of coupled integro-differential equations for the functions $\chi_{k}\left(\overline{\mathrm{R}}_{k}\right)$.

To see this more explicitly, consider again the special case given in Eq. (7). Then the last term in Eq. (10) will lead to

$$
-E \int_{\text {fixed } \overrightarrow{\mathbf{R}}_{A}} \phi_{1 s_{A}}\left(\overrightarrow{\mathrm{r}}_{A}\right) \chi_{2}\left(\overrightarrow{\mathrm{R}}_{B}\right) \phi_{1 s_{B}}\left(\overrightarrow{\mathrm{r}}_{B}\right) d \overrightarrow{\mathrm{r}}_{A}=-E \int_{\text {fixed } \overrightarrow{\mathrm{r}}_{A}} \phi_{1 s_{A}}\left(\overrightarrow{\mathrm{r}}_{A}\right) \chi_{2}\left(\overrightarrow{\mathrm{R}}_{B}\left(\overrightarrow{\mathrm{r}}_{A}, \overrightarrow{\mathrm{R}}_{A}\right)\right) \phi_{1 s_{B}}\left(\overrightarrow{\mathrm{r}}_{B}\left(\overrightarrow{\mathrm{r}}_{A}, \overrightarrow{\mathrm{R}}_{A}\right)\right) d \overrightarrow{\mathrm{r}}_{A},
$$

where, in the second line, $\vec{r}_{B}$ and $\vec{R}_{B}$ have been eliminated in favor of $\vec{r}_{A}, \vec{R}_{A}$. Other terms are handled similarly.

We may note already that no displacement couplings appear in these equations. The derivative $\vec{\nabla}_{R_{k}} \phi_{k}$ is evaluated with $\vec{r}_{k}$ held fixed, so it represents the rate of change of $\phi_{k}$ as seen in a frame that is moving with the center to which $\phi_{k}$ is attached. Such change of $\phi_{k}$ represents only rotation or other parametric variation of $\phi_{k}$, and this type of change leads to real nonadiabatic coupling. The "fictitious" displacement couplings arise in other formulations when one takes, for example, $\vec{\nabla}_{k} \phi_{k}\left(\overrightarrow{\mathrm{r}}_{k}\right)$ at fixed $\overrightarrow{\mathrm{r}}$, or, more generally, $\vec{\nabla}_{R_{j}} \phi_{k}\left(\overrightarrow{\mathrm{r}}_{k}\right)$ at fixed $\overrightarrow{\mathrm{r}}_{j}$.

\section{APPROXIMATIONS SUITABLE FOR SLOW ATOMIC COLLISIONS}

The coupled integro-differential equations (12) are "exact", in the sense that they follow from the ansatz (6) without approximations. In principle, it should be possible to solve them by an iterative self-consistent method, but such an approach would be unnecessarily tedious and difficult. It is more sensible to look for simplifying approximations. Mott and Massey ${ }^{10}$ show how to make a kind of distorted-wave approximation, but this method is only suitable for systems having weak coupling. Here we present approximations which are valid very generally for slow atomic collisions. We will make use of the fact that the nuclear masses are much larger than the electron mass, and we will assume that the relative velocity of the nuclei is small compared to typical electron velocities.

Let us begin by transforming into mass-scaled coordinates, defined as in (3),

$$
\begin{aligned}
& \overrightarrow{\mathbf{r}}_{k}^{m}=m_{k}^{1 / 2} \overrightarrow{\mathbf{r}}_{k}, \\
& \overrightarrow{\mathrm{R}}_{k}^{m}=\mu_{k}^{1 / 2} \overrightarrow{\mathbf{R}}_{k} .
\end{aligned}
$$

A function or operator marked with a superscript $m$, as $F^{m}$ or $F^{m}\left(\overrightarrow{\mathrm{r}}_{k}^{m}, \overrightarrow{\mathbf{R}}_{k}^{m}\right)$, is the reexpression of the function or operator $F\left(\overrightarrow{\mathrm{r}}_{k}, \overrightarrow{\mathrm{R}}_{k}\right)$ in terms of massscaled coordinates: For example,

$$
\begin{aligned}
& \phi_{k}^{m}\left(\overrightarrow{\mathrm{r}}_{k}^{m} ; \overrightarrow{\mathrm{R}}_{k}^{m}\right)=\phi_{k}\left(\overrightarrow{\mathrm{r}}_{k}, \overrightarrow{\mathrm{R}}_{k}\right), \\
& \vec{\nabla}_{R_{k}}^{m}=\vec{\nabla}_{R_{k}^{m}}=\left(\mu_{k}\right)^{-1 / 2} \vec{\nabla}_{R_{k}} .
\end{aligned}
$$

Thus we have

$$
\begin{aligned}
\mathcal{O}=\sum_{k} & \left\{-\frac{1}{2} \hbar^{2}\left[\phi_{k}^{m}\left(\nabla_{R_{k}}^{m}\right)^{2} \chi_{k}^{m}+2 \vec{\nabla}_{R_{k}}^{m} \phi_{k}^{m} \cdot \vec{\nabla}_{R_{k}}^{m} \chi_{k}^{m}\right.\right. \\
& \left.\left.+\chi_{k}^{m}\left(\nabla_{R_{k}}^{m}\right)^{2} \phi_{k}^{m}\right]+\chi_{k}^{m} h_{k}^{m} \phi_{k}^{m}-E \chi_{k}^{m} \phi_{k}^{m}\right\}
\end{aligned}
$$

and

$$
\int_{\text {fixed } \overrightarrow{\mathrm{R}}_{j}^{m}}\left[\phi_{j}^{m}\left(\overrightarrow{\mathrm{r}}_{j}^{m} ; \overrightarrow{\mathrm{R}}_{j}^{m}\right)\right] * \Theta_{j}^{m}\left(\overrightarrow{\mathrm{r}}_{j}^{m}, \overrightarrow{\mathrm{R}}_{j}^{m}\right) m_{j}^{1 / 2} d \overrightarrow{\mathbf{r}}_{j}^{m}=0
$$

As mentioned earlier, after each term in (15) is evaluated, it has to be reexpressed as a function of $\left(\overrightarrow{\mathbf{r}}_{j}^{m}, \overrightarrow{\mathrm{R}}_{j}^{m}\right)$ before carrying out the integration (16). Most of the quantities appearing in (15), [in particular $\phi_{k}^{m}, h_{k}^{m} \phi_{k}^{m}, \nabla_{R_{k}}^{m} \phi_{k}^{m}$, and $\left.\left(\nabla_{R_{k}}^{m}\right)^{2} \phi_{k}^{m}\right]$, can be evaluated in closed form in their original coordinates $\left(\overrightarrow{\mathrm{r}}_{k}^{m}, \overrightarrow{\mathrm{R}}_{k}^{m}\right)$. Hence their reexpression in terms of $\left(\vec{r}_{j}^{m}, \vec{R}_{j}^{m}\right)$ is a simple matter, because there is a linear relationship between various pairs of coordinates :Table I). 
This cannot be done for $\chi_{k}^{m}$ or its derivatives, however, because their functional forms are not determined until after the equations are solved. Hence for $\chi_{k}^{m}$ we use the approximation

$$
\begin{aligned}
\chi_{k}^{m}\left(\overrightarrow{\mathrm{R}}_{k}^{m}\right) & =\chi_{k}^{m}\left(\overrightarrow{\mathrm{R}}_{k}^{m}\left(\overrightarrow{\mathbf{r}}_{j}^{m}, \overrightarrow{\mathrm{R}}_{j}^{m}\right)\right) \\
& =\chi_{k}^{m}\left(\overrightarrow{\mathrm{R}}_{j}^{m}+\overrightarrow{\mathrm{R}}_{k}^{m}\left(\overrightarrow{\mathbf{r}}_{j}^{m}, \overrightarrow{\mathbf{R}}_{j}^{m}\right)-\overrightarrow{\mathbf{R}}_{j}^{m}\right) \\
& \simeq \chi_{k}^{m}\left(\overrightarrow{\mathbf{R}}_{j}^{m}\right)+\left(\overrightarrow{\mathbf{s}}_{k}^{m}-\overrightarrow{\mathbf{s}}_{j}^{m}\right) \cdot \vec{\nabla}_{R_{j}}^{m} \chi_{k}^{m}\left(\overrightarrow{\mathbf{R}}_{j}^{m}\right),
\end{aligned}
$$

where

$$
\overrightarrow{\mathbf{S}}_{k}^{m}=\overrightarrow{\mathrm{R}}_{k}^{m}-\overrightarrow{\mathrm{R}}^{m} .
$$

Approximation (17) is appropriate if higher terms in the Taylor expansion are negligible: Essentially, we must have $\left(\overrightarrow{\mathbf{s}}_{k}^{m}-\overrightarrow{\mathbf{s}}_{j}^{m}\right)$ small compared to the wavelength of oscillations of $\chi_{k}\left(\vec{R}_{j}^{m}\right)$. The physical meaning is more transparent in unscaled coordinates: defining

$$
\overrightarrow{\mathbf{s}}_{k}=\left(\mu^{1 / 2} / m\right) \overrightarrow{\mathbf{s}}_{k}^{m},
$$

it follows that

$$
(m / \mu) \overrightarrow{\mathbf{S}}_{k}=\left[\left(\mu_{k} / \mu\right)^{1 / 2} \overrightarrow{\mathbf{R}}_{k}-\overrightarrow{\mathbf{R}}\right]
$$

and the second term in (17) is $(m / \mu)\left(\overrightarrow{\mathbf{S}}_{k}-\overrightarrow{\mathbf{s}}_{j}\right) \cdot \vec{\nabla}_{R_{k}} \chi_{k}\left(\overrightarrow{\mathbf{R}}_{k}\right)$. Now $\left|\vec{\nabla}_{R_{k}} \chi_{k}\right|$ is of the order $\lambda_{\mathrm{deB}}^{-1}\left|\chi_{k}\right|$, where $\lambda_{\mathrm{deB}}$ is the de Broglie wavelength associated with the relative nuclear motion. The magnitude of $\left(\vec{s}_{k}-\vec{s}_{j}\right)$ is of the order of atomic dimensions, $a_{0}$. Thus the second term in (17) will be small compared to the first term if

$$
(m / \mu)\left(a_{0} / \lambda_{\text {deB }}\right) \ll 1 \text {. }
$$

In a typical atomic collision, the de Broglie wavelength is small compared to atomic dimensions, but in (20a), $\lambda_{\text {deB }}$ is compared with $(m / \mu) a_{0}$. This length is comparable to the distance from the nucleus to the center of mass of the atom, and (17) is appropriate if $\lambda_{\mathrm{deB}}$ is large compared to this distance. Equivalently, taking $2 \pi \hbar / \mu \lambda_{\text {deB }}=v_{\text {nuc }}$ and $m a_{0} \sim 2 \pi \hbar / v_{e l}$, condition (20a) is simply

$$
\left(v_{\text {nuc }} / v_{e l}\right) \ll 1 \text {. }
$$

By. means of approximation (17), the integrodifferential equations (12) can be reduced to coupled second-order differential equations. For example, the last term in (15) becomes

$-E \int\left(\phi_{j}^{m}\right)^{*} \phi_{k}^{m} m_{j}^{1 / 2} d \overrightarrow{\mathbf{r}}_{j}^{m} \chi_{k}^{m}\left(\overrightarrow{\mathbf{R}}_{j}^{m}\right)-E \int\left(\phi_{j}^{m}\right) *\left(\overrightarrow{\mathbf{S}}_{k}^{m}-\overrightarrow{\mathbf{S}}_{j}^{m}\right) \phi_{k}^{m} m_{j}^{1 / 2} d \overrightarrow{\mathbf{r}}_{j}^{m} \cdot \vec{\nabla}_{R_{j}}^{m} \vec{\chi}_{k}^{m}\left(\overrightarrow{\mathbf{R}}_{j}^{m}\right)=-E S_{j k}^{m} \chi_{k}^{m}\left(\overrightarrow{\mathbf{R}}_{j}^{m}\right)-i \hbar \vec{\sigma}_{j k}^{m} \cdot \vec{\nabla}_{R_{j}}^{m} \chi_{k}^{m}\left(\overrightarrow{\mathrm{R}}_{j}^{m}\right)$

[notation defined in Eq. (23)]. Similarly, evaluating all terms in (15), we obtain the coupled equations

$$
\begin{aligned}
& \left\{\left(\underline{S}^{m}-i \hbar \underline{\vec{\sigma}}^{m} \cdot \vec{\nabla}^{m}\right)\left[-\frac{1}{2} \hbar^{2}\left(\nabla^{m}\right)^{2}\right]+\left(-i \hbar \underline{\vec{\Pi}}^{m} \cdot \vec{\nabla}^{m}-\hbar^{2} \underline{\pi}^{m}: \vec{\nabla}^{m} \vec{\nabla}^{m}\right)\right. \\
& \left.\quad+\frac{1}{2}\left(\underline{B}^{m}-i \hbar \underline{\vec{\beta}}^{m} \cdot \vec{\nabla}{ }^{m}\right)+\left(\underline{h}^{m}-i \hbar \underline{\vec{\eta}}^{m} \cdot \vec{\nabla} \underline{\nabla}^{m}\right)-E\left(\underline{S}^{m}-i \hbar \underline{\vec{\sigma}}^{m} \cdot \vec{\nabla}{ }^{m}\right)\right\} \underline{\chi}^{m}=0,
\end{aligned}
$$

where

$$
\begin{aligned}
& S_{j k}^{m}=\int\left(\phi_{j}^{m}\right)^{*} \phi_{k}^{m} m_{j}^{1 / 2} d \overrightarrow{\mathrm{r}}_{j}^{m}, \\
& \vec{\sigma}_{j^{k}}^{m}=\frac{i}{\hbar} \int\left(\phi_{j}^{m}\right) *\left(\overrightarrow{\mathbf{S}}_{k}^{m}-\overrightarrow{\mathbf{s}}_{j}^{m}\right) \phi_{k}^{m} m_{j}^{1 / 2} d \overrightarrow{\mathbf{r}}_{j}^{m}, \\
& \vec{\Pi}_{j^{k}}^{m}=\int\left(\phi_{j}^{m}\right) *\left(-i \hbar \vec{\nabla}_{R_{k}}^{m} \phi_{k}^{m}\right)_{r_{k}} m_{j}^{1 / 2} d \overrightarrow{\mathbf{r}}_{j}^{m}, \\
& \ddot{\pi}_{j^{k}}^{m}=\int\left(\phi_{j}^{m}\right) *\left(\overline{\mathbf{S}}_{k}^{m}-\overleftarrow{\mathbf{S}}_{j}^{m}\right)\left(\vec{\nabla}_{R_{k}}^{m} \phi_{k}^{m}\right)_{r_{k}} m_{j}^{1 / 2} d \overrightarrow{\mathrm{r}}_{j}^{m}, \\
& B_{j^{k}}^{m}=\int\left(\phi_{j}^{m}\right) *\left[-\hbar^{2}\left(\nabla_{R_{k}}^{m}\right)^{2} \phi_{k}^{m}\right]_{r_{k}} m_{j}^{1 / 2} d \overrightarrow{\mathrm{r}}_{j}^{m}, \\
& \vec{\beta}_{j^{k}}^{m}=-i \hbar \int\left(\phi_{j}^{m}\right) *\left(\overrightarrow{\mathbf{S}}_{k}^{m}-\overrightarrow{\mathrm{S}}_{j}^{m}\right)\left[\left(\nabla_{R_{k}}^{m}\right)^{2} \phi_{k}^{m}\right]_{r_{k}} m_{j}^{1 / 2} d \overrightarrow{\mathrm{r}}_{j}^{m}, \\
& h_{j k}^{m}=\int\left(\phi_{j}^{m}\right) * h_{k}^{m} \phi_{k}^{m} m_{j}^{1 / 2} d \overrightarrow{\mathrm{r}}_{j}^{m}, \\
& \vec{\eta}_{j^{k}}^{m}=(i / \hbar) \int\left(\phi_{j}^{m}\right) *\left(\overrightarrow{\mathbf{S}}_{k}^{m}-\overrightarrow{\mathbf{s}}_{j}^{m}\right) h_{k}^{m} \phi_{k}^{m} m_{j}^{1 / 2} d \overrightarrow{\mathrm{r}}_{j}^{m} .
\end{aligned}
$$

In Eqs. (23c)-(23f) the derivatives $\nabla_{R_{k}}^{m}$ are to be evaluated at fixed $\overrightarrow{\mathrm{r}}_{k}^{m}$; in Eq. $(23 \mathrm{~h}) h_{k}^{m}$ contains $\left(\nabla_{\boldsymbol{r}_{k}}^{m}\right)^{2}$, which is to be evaluated at fixed $R_{k}^{m}$. Then everything inside the integrals has to be reexpressed as functions of $\left(\overrightarrow{\mathrm{r}}_{j}^{m}, \overrightarrow{\mathrm{R}}_{j}^{m}\right)$ and integration is performed holding $\overrightarrow{\mathbf{R}}_{j}^{m}$ fixed. The independent variable in the $j$ th equation of the set (22) is $\vec{R}_{f}^{m}$, and $\vec{\nabla}^{m}$ means $\vec{\nabla}_{R_{j}}^{m}$.

Because of the term $\vec{\sigma}^{m} \cdot \vec{\nabla}^{m \frac{1}{2}}\left(-i \hbar \nabla^{m}\right)^{2} \underline{\chi}$, these differential equations are of third order. However, they can be reduced to second order by an approximation that is consistent with (17): Multiplying (22) by

$$
\underline{S}^{m}\left(\underline{S}^{m}-i \hbar \underline{\vec{\sigma}}^{m} \cdot \vec{\nabla}^{m}\right)^{-1} \simeq \underline{1}+i \hbar \underline{\vec{\sigma}}^{m} \cdot \vec{\nabla}^{m}\left(\underline{S}^{m}\right)^{-1}
$$

and dropping terms such as $\sigma^{m} \pi^{m}, \sigma^{m} \beta^{m}$, and $\sigma^{m} \underline{\eta}^{m}$ [which are related to the negīected higher-order terms in the Taylor expansion (17)], we obtain

$$
\begin{aligned}
& \left\{\frac{1}{2} \underline{S}^{m}\left(-i \hbar \nabla^{m}\right)^{2}+\left[1+i \hbar \underline{\vec{\sigma}}^{m} \cdot \vec{\nabla}^{m}\left(\underline{S}^{m}\right)^{-1}\right]\right. \\
& \times\left(\underline{h}^{m}-i \hbar \underline{\vec{T}}^{m} \cdot \vec{\nabla}^{m}+\frac{1}{2} \underline{B}^{m}\right)+-i \hbar\left(\underline{\vec{\eta}}^{m} \cdot \vec{\nabla}^{m}+\frac{1}{2} \vec{\beta}^{m} \cdot \vec{\nabla}\right. \\
& \\
& \left.-\hbar^{2} \underline{\vec{\pi}}^{m}: \vec{\nabla}^{m} \vec{\nabla}^{m}-E \underline{S}^{m}\right\} \underline{\chi}^{m}=0 .
\end{aligned}
$$


The next step is either very obvious or very subtle. Equations (25) can be written more abstractly in the form

$$
\sum_{k} M_{j k}\left(\overrightarrow{\mathrm{R}}_{j}^{m}\right) \chi_{k}\left(\overrightarrow{\mathrm{R}}_{j}^{m}\right)=0,
$$

where $M_{j k}$ is a matrix containing functions of $\overrightarrow{\mathrm{R}}_{j}^{m}$ and derivatives with respect to $\overrightarrow{\mathrm{R}}_{j}^{m}$. In the development up to this point, the various coordinates $\overrightarrow{\mathrm{R}}_{j}^{m}$ are distinct, being $\overrightarrow{\mathrm{R}}_{A}^{m}, \overrightarrow{\mathrm{R}}_{B}^{m}, \overrightarrow{\mathrm{R}}^{m}$, etc., depending on the center with which the basis function $\phi_{j}$ is associated. However, for the purpose of integration of the coupled equations (26a), the various coordinates $\vec{R}_{j}^{m}$ all play the same role: They are all dummy integration variables, and in any form of step-by-step integration of (26a), they would all be set numerically equal to each other. In other words, equations of the form (26a) are entirely equivalent to the equations

$$
\sum_{k} M_{j k}\left\langle\overrightarrow{\mathrm{R}}^{m}\right\rangle \chi_{k}\left\langle\overrightarrow{\mathrm{R}}^{m}\right\rangle=0
$$

where $\left\langle\overrightarrow{\mathrm{R}}^{m}\right\rangle$ represents the common numerical value of the coordinates $\overrightarrow{\mathrm{R}}_{j}^{m}$. (If further explanation of this step is needed, see Appendix C.)

Now it is convenient to transform back out of mass-scaled coordinates by defining

$$
\langle\overrightarrow{\mathrm{R}}\rangle=\left\langle\overrightarrow{\mathrm{R}}^{m}\right\rangle / \mu^{1 / 2} \text {. }
$$

Then the coupled equations (25) take the form

$$
\begin{aligned}
& \left\{(1 / 2 \mu) \underline{S}(-i \hbar \nabla)^{2}+\left[1+(i \hbar / \mu) \underline{\vec{\sigma}} \cdot \vec{\nabla} \underline{S}^{-1}\right]\right. \\
& {[\underline{h}-(i \hbar / \mu) \underline{\vec{\Pi}} \cdot \vec{\nabla}+(1 / 2 \mu) \underline{B}]+(1 / \mu)\left[\left(\vec{\eta}+\frac{1}{2} \vec{\beta}\right) \cdot(-i \hbar \vec{\nabla})\right.} \\
& +\pi \pi:(-i \hbar \bar{\nabla})(-i \hbar \vec{\nabla})]-\underline{S} E\} \underline{\chi}=0 \text {. }
\end{aligned}
$$

The independent variable in all equations is $\langle\vec{R}\rangle$, $\vec{\nabla}$ means $\vec{\nabla}_{\langle\vec{R}\rangle}$, and all matrices are defined in Table III. The $\vec{\nabla}$ in the term $\vec{\sigma} \cdot \vec{\nabla}$ acts on everything to the right, including $S^{-1}, h, x$, etc.

Equations (28) provide a tractable set of coupled equations, obtained from (12) by only two approximations [truncation of the Taylor series (17) and truncation of the inverse-operator series (24)], both of which are valid under the condition (20). However, the same condition (20) permits further simplification of the equations by the following method. When $(-i \hbar \vec{\nabla})$ acts on $\underline{\chi}$, the magnitude of the result is of order $\mu v_{\text {nuc }}|\chi|$, but when it acts on any other quantity $Q$ (including $\phi_{k}, \underline{S}^{-1}, \underline{h}, \vec{\Pi}, \underline{B}$ ) the magnitude of the result is of order $\left(\hbar / a_{0}\right) \mid Q$ $=m v_{e l}|Q|$. Let us assume that the nuclear kinetic energy $\mu v_{\text {nuc }}^{2} / 2$, the energies of the significant electronic states $|h|$, and the total energy $E$ are all of the same order of magnitude, which we call "zeroth order" in $\left(v_{\text {nuc }} / v_{\text {el }}\right)$. It follows that $\left(v_{\text {nuc }} / v_{\text {el }}\right) \sim(m / \mu)^{1 / 2}$. Then the various terms in (28) can be sorted in powers of $\left(v_{\text {nuc }} / v_{\text {el }}\right)$ or $(m / \mu)^{1 / 2}$ as follows.

zeroth order:

TABLE III. Coupling matrices. $\phi_{k}=\phi_{k}\left(\overrightarrow{\mathrm{r}}_{k} ; \overrightarrow{\mathrm{R}}_{k}\right) . \vec{\nabla} \phi_{k}=\left[\vec{\nabla}_{R_{h}} \phi\left(\overrightarrow{\mathrm{r}}_{k} ; \overrightarrow{\mathrm{R}}_{k}\right)\right]_{\overrightarrow{\mathrm{r}}_{X}} \cdot h_{k}$ defined in Eq. (9b). After differentiation, all quantities must be written as functions of $\overrightarrow{\mathrm{r}}_{j}$ and $\overrightarrow{\mathrm{R}}_{j}$, and integration is performed holding $\overrightarrow{\mathrm{R}}_{j}$ fixed. $\kappa_{k}=\frac{1}{2}\left(f_{k}+\lambda\right) ; f_{B}=1, f_{A}=-1$.

\begin{tabular}{lll}
\hline \hline$S_{j k}=\int \phi_{j}^{*} \phi_{k} d \overrightarrow{\mathrm{r}}_{j}$ & overlap \\
$h_{j k}=\int \phi_{j}^{*} h_{k} \phi_{k} d \overrightarrow{\mathrm{r}}_{j}$ & electronic Hamiltonian \\
$\overrightarrow{\mathrm{\Pi}}_{j k}=\int \phi_{j}^{*}-i \hbar \vec{\nabla} \phi_{k} d \overrightarrow{\mathrm{r}}_{j}$ & vector nonadiabatic coupling \\
$B_{j k}=-\hbar^{2} \int \phi_{j}^{*} \nabla^{2} \phi_{k} d \overrightarrow{\mathrm{r}}_{j}$ & second derivative \\
$\vec{\gamma}=\underline{\underline{\eta}}-\underline{\vec{\sigma}} \underline{S}^{-1} \underline{h}$ & momentum transfer \\
$\vec{\sigma}_{j k}=\frac{i m}{\hbar} \int \phi_{j}^{*}\left(\overrightarrow{\mathrm{s}}_{k}-\overrightarrow{\mathrm{s}}_{j}\right) \phi_{k} d \overrightarrow{\mathrm{r}}_{j}$ & \\
$\vec{\eta}_{j k}=\frac{i m}{\hbar} \int \phi_{j}^{*}\left(\overrightarrow{\mathrm{s}}_{k}-\overrightarrow{\mathrm{s}}_{j}\right) h_{k} \phi_{k} d \overrightarrow{\mathrm{r}}_{j}$ & \\
$\vec{\pi}_{j k}=\frac{m}{\mu} \int \phi_{j}^{*}\left(\overrightarrow{\mathrm{s}}_{k}-\overrightarrow{\mathrm{s}}_{j}\right) \vec{\nabla}_{\phi_{k}} d \overrightarrow{\mathrm{r}}_{j}$ & \\
$\vec{\beta}_{j k}=\frac{i \hbar m}{\mu} \int \phi_{j}^{*}\left(\overrightarrow{\mathrm{s}}_{k}-\overrightarrow{\mathrm{s}}_{j}\right) \nabla^{2} \phi_{k} d \overrightarrow{\mathrm{r}}_{j}$ & \\
$\overrightarrow{\mathrm{s}}_{k}=(\mu / m)\left[\left(\mu_{k} / \mu\right)^{1 / 2} \overrightarrow{\mathrm{R}}_{k}-\overrightarrow{\mathrm{R}}\right]$ & \\
$\simeq$ & $\simeq \kappa_{k} \overrightarrow{\mathrm{r}}-\frac{1}{2} \kappa_{k}^{2} \overrightarrow{\mathrm{R}}$
\end{tabular}


$(2 \mu)^{-1} \underline{S}(-i \hbar \nabla)^{2} \underline{\chi}, \underline{h} \underline{\chi}, E \underline{\chi}$,

first order:

$$
\mu^{-1} \underline{\vec{\Pi}} \cdot(-i \hbar \vec{\nabla} \underline{\chi}), \quad \mu^{-1} \underline{\vec{\eta}} \cdot(-i \hbar \vec{\nabla} \underline{\chi}), \quad \mu^{-1} \underline{\underline{\sigma}} \underline{S}^{-1} \underline{h} \cdot \vec{\nabla} \underline{\chi},
$$

second order: everything else except third order,

third order:

$$
\underline{\chi} \mu^{-2} \underline{\vec{\sigma}} \cdot\left(\vec{\nabla} \underline{S}^{-1} \underline{B}\right) \text {. }
$$

In the earlier approximations [(17) and (24)], all terms that are second order (or higher) in $v_{\text {nuc }} / v_{e l}$ and which are related to $\left(\overrightarrow{\mathbf{s}}_{k}-\overrightarrow{\mathbf{s}}_{j}\right)$ were neglected. Therefore, without significant loss of accuracy, all such second-order terms in (28) can also be neglected. The matrix $B$ is also second-order in $\left(v_{\text {nuc }} / v_{e l}\right)$, but it is not related to $\left(\overrightarrow{\mathbf{s}}_{k}-\overrightarrow{\mathbf{s}}_{j}\right)$; it can be neglected or retained, and we will retain it in the formal development below. ${ }^{14}$ After small terms are discarded, the equations reduce to the final simplified form

$$
\begin{aligned}
& {\left[(2 \mu)^{-1} \underline{S}(-i \hbar \nabla)^{2}+\underline{h}-\underline{S} E+\mu^{-1}(\underline{\vec{\Pi}}+\underline{\vec{\gamma}}) \cdot(-i \hbar \vec{\nabla})\right.} \\
& \left.+(2 \mu)^{-1} \underline{B}\right] \underline{\chi}=0 .
\end{aligned}
$$

Coupling matrices appearing here are defined in Table III.

\section{EVALUATION OF ELEMENTARY MATRIX ELEMENTS}

Let us examine more carefully the matrix elements that appear in Eqs. (29). All of the matrices are similar to matrices that are familiar in molecular structure calculations, but the present theory introduces some significant modifications.

\section{A. Overlap matrix, $S$}

The matrix $S$ represents the overlap between various nonorthogonal basis functions, but it differs by terms of order $(m / \mu)$ from the conventional overlap matrix. As an example, let us consider again the special case (7). In atomic units $\left(m_{0}=1, a_{0}=1, \hbar=1\right)$ one usually writes

$$
\begin{aligned}
& \phi_{1 s_{A}}=N_{A}^{\prime \prime} \exp \left(-\left|\overrightarrow{\mathrm{r}}_{A}\right|\right), \\
& \phi_{1 s_{B}}=N_{B}^{\prime \prime} \exp \left(-\left|\overrightarrow{\mathrm{r}}_{B}\right|\right),
\end{aligned}
$$

but if we want the basis functions to satisfy the boundary conditions exactly, we should write

$$
\begin{aligned}
& \phi_{1 s_{A}}=N_{A} \exp \left(-m_{A}\left|\overrightarrow{\mathrm{r}}_{A}\right|\right), \\
& \phi_{1 s_{B}}=N_{B} \exp \left(-m_{B}\left|\overrightarrow{\mathrm{r}}_{B}\right|\right) .
\end{aligned}
$$

Evaluation of the overlap then proceeds according to the instructions given below Eq. (15): The element $S_{1 s_{A} 1 s_{B}}$ is in principle a function of $\vec{R}_{A}$, so we have to reexpress $\phi_{1 s_{B}}$ in terms of $\left(\overrightarrow{\mathrm{r}}_{A}, \overrightarrow{\mathrm{R}}_{A}{ }^{A}\right)$. (Transformation into and back out of mass-scaled coordinates does not affect the matrix elements. ${ }^{15}$ ) From Fig. 1,

$$
\overrightarrow{\mathbf{r}}_{B}=\frac{M_{A}}{M_{A}+m_{0}} \overrightarrow{\mathbf{r}}_{A}-\overrightarrow{\mathrm{R}}_{A},
$$

so

$$
\begin{aligned}
S_{1 s_{A} 1 s_{B}}\left(\overrightarrow{\mathrm{R}}_{A}\right)=N_{A} N_{B} \int & \exp \left(-m_{A}\left|\overrightarrow{\mathrm{r}}_{A}\right|\right) \\
& \times \exp \left(-m_{B}\left|\frac{M_{A}}{M_{A}+m_{0}} \overrightarrow{\mathrm{r}}_{A}-\overrightarrow{\mathrm{R}}_{A}\right|\right) d \overrightarrow{\mathrm{r}}_{A} .
\end{aligned}
$$

Finally, as in the change from Eq. (26a) to (26b), we replace $\vec{R}_{A}$ by $\langle\vec{R}\rangle$ on both sides of this expression. The result differs from the more conventional overlap

$$
\begin{aligned}
S_{1 s_{A} 1 s_{B}}^{\prime}=N_{A} N_{B} \int & \exp \left(-m_{A}\left|\overrightarrow{\mathrm{r}}_{A}\right|\right) \\
& \times \exp \left(-m_{B}\left|\overrightarrow{\mathrm{r}}_{A}-\overrightarrow{\mathrm{R}}\right|\right) d \overrightarrow{\mathrm{r}}_{A},
\end{aligned}
$$

or the still more simplified

$$
S_{1 s_{A} 1 s_{B}}^{\prime \prime}=N_{A}^{\prime \prime} N_{B}^{\prime \prime} \int \exp \left(-\left|\overrightarrow{\mathrm{r}}_{A}\right|\right) \exp \left(-\left|\overrightarrow{\mathrm{r}}_{A}-\overrightarrow{\mathrm{R}}\right|\right) d \overrightarrow{\mathrm{r}}_{A},
$$

by terms of order $(m / \mu)$. For most practical purposes this difference is insignificant. In the first place, very few calculations on real collision systems are carried out to such a high level of accuracy, and in the second place, the difference between (32a), (32b), and (32c) is as small as terms that have already been neglected. Hence it is safe to regard $S$ as being an ordinary overlap matrix.

\section{B. Electronic Hamiltonian matrix, $h$}

The matrix representing the electronic Hamiltonian also differs from more conventional forms by terms of order $(m / \mu)$. These differences arise from three sources. The first is the electronic kinetic energy operator, $-\hbar^{2} \nabla_{r_{k}}^{2} / 2 m_{k}$, which contains the atomic reduced mass, $m_{A}$ or $m_{B}$, instead of the molecular reduced mass, $m$. The other two sources are related to the transformation from $\left(\overrightarrow{\mathrm{r}}_{k}, \overrightarrow{\mathrm{R}}_{k}\right)$ to $\left(\overrightarrow{\mathrm{r}}_{j}, \overrightarrow{\mathrm{R}}_{j}\right):$ The basis functions are transformed as in Eq. (32a), but also the potential energy is transformed in the same way. For example, the matrix element

$$
\int \phi_{1 s_{A}}^{*}\left|\overrightarrow{\mathrm{r}}_{B}\right|^{-1} \phi_{1 s_{B}} d r_{A}
$$

becomes

$$
\begin{aligned}
N_{A} N_{B} \int & \exp \left(-m_{A}\left|\overrightarrow{\mathrm{r}}_{A}\right|\right)\left|\frac{M_{A}}{M_{A}^{\prime}+m_{0}} \overrightarrow{\mathrm{r}}_{A}-\langle\overrightarrow{\mathrm{R}}\rangle\right|^{-1} \\
& \times \exp \left(-m_{B}\left|\frac{M_{A}}{M_{A}+m_{0}} \overrightarrow{\mathrm{r}}_{A}-\langle\overrightarrow{\mathrm{R}}\rangle\right|\right) d \overrightarrow{\mathrm{r}}_{A}
\end{aligned}
$$


when expressed as a function of $\langle\vec{R}\rangle$. Again the difference between this form and the more conventional one

$$
\begin{aligned}
N_{A} N_{B} \int & \exp \left(-m_{A}\left|\overrightarrow{\mathrm{r}}_{A}\right|\right)\left|\overrightarrow{\mathrm{r}}_{A}-\overrightarrow{\mathrm{R}}\right|^{-1} \\
& \times \exp \left(-m_{B}\left|\overrightarrow{\mathrm{r}}_{A}-\overrightarrow{\mathrm{R}}\right|\right) d \overrightarrow{\mathrm{r}}_{A}
\end{aligned}
$$

is negligible for most practical purposes. Similar considerations apply to all the other matrix elements, but we will ignore them henceforth.

Note that in the evaluation of matrix elements, neglect of these small corrections is equivalent to neglecting the differences between $\overrightarrow{\mathrm{R}}_{A}, \overrightarrow{\mathrm{R}}_{B}, \overrightarrow{\mathrm{R}}$, and $\langle\overrightarrow{\mathrm{R}}\rangle$. Such neglect is justified at this point in the calculation, but not earlier.

\section{Nonadiabatic coupling matrices $\underline{\vec{I}}$ and $\underline{B}$}

The vector nonadiabatic coupling matrix $\vec{\Pi}$ can be separated into "radial" and "angular" components in the usual way ${ }^{1,3(a)}$ and we will discuss these components separately.

\section{Separation of radial and angular components}

Like all the other matrices, $\overrightarrow{\mathbb{I}}$ is a function of the vector $\langle\vec{R}\rangle$. That vector specifies a direction in space, and we can use it to select components of $\vec{I}$ and also to define a frame of reference for describing the position of the electron. We have taken $(x, y, z)$ to represent the components of the vector $\overrightarrow{\mathrm{r}}$ relative to an external "space-fixed" frame of reference. Relative to this same spacefixed frame, the vector $\langle\overrightarrow{\mathrm{R}}\rangle$ can be specified by its length $\langle R\rangle$ and two spherical-polar angles, $\theta$, $\Phi$. Then the "rotating" frame of reference is defined by using these polar angles as Euler angles (Fig. 3). It follows that, for any vector $\overrightarrow{\mathrm{u}}$, the components $\left(u_{x}^{\prime}, u_{y}^{\prime}, u_{z}^{\prime}\right)$ along the rotating axes are related to components on space-fixed axes by

$$
\left[\begin{array}{c}
u_{x}^{\prime} \\
u_{y}^{\prime} \\
u_{z}^{\prime}
\end{array}\right]=\left[\begin{array}{ccc}
\cos \Theta \cos \Phi & \cos \Theta \sin \Phi & -\sin \Theta \\
-\sin \Theta & \cos \Theta & 0 \\
\sin \Theta \cos \Phi & \sin \Theta \sin \Phi & \cos \Theta
\end{array}\right]\left[\begin{array}{l}
u_{x} \\
u_{y} \\
u_{z}
\end{array}\right] .
$$

Of course, the same transformation applies to the components of any vector, so, for the vector $\overrightarrow{\mathrm{r}}_{A}$, for example, (34) describes the relationship between space-fixed components $\left(x_{A}, y_{A}, z_{A}\right)$ and rotating components $\left(x_{A}^{\prime}, y_{A}^{\prime}, z_{A}^{\prime}\right)$.

$\vec{\Pi}$ contains the gradient $\vec{\nabla}_{\langle R\rangle}$, and it is convenient to express this operator in polar components,

$$
\vec{\nabla}_{\langle R\rangle}=\left(\frac{\partial}{\partial\langle R\rangle},\langle R\rangle^{-1} \frac{\partial}{\partial \theta},(\langle R\rangle \sin \Theta)^{-1} \frac{\partial}{\partial \Phi}\right) .
$$

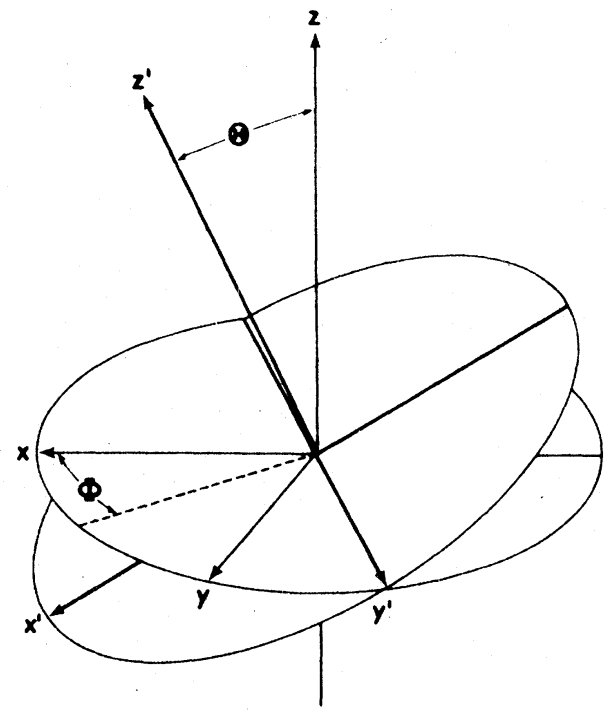

FIG. 3. Space-fixed and molecule-fixed reference frames for the molecular electron. $(x, y, z)$ denote space-fixed axes, $\left(x^{\prime}, y^{\prime}, z^{\prime}\right)$, molecule-fixed axes, and they are related via rotations by angles $(\theta, \phi)$ as shown.

Then, using (34), one can show that

$$
\begin{aligned}
& \left(-i \hbar \frac{\partial}{\partial\langle R\rangle}\right)_{x_{k} y_{k}^{z} z_{k}}=-i \hbar\left(\frac{\partial}{\partial\langle R\rangle}\right)_{x_{k}^{\prime} y_{k}^{\prime} z_{k}^{\prime}}, \\
& \left(-i \hbar \frac{\partial}{\partial \Theta}\right)_{x_{k} y_{k}^{z} z_{k}}=\left(-i \hbar \frac{\partial}{\partial \Theta}\right)_{x_{k}^{\prime} y_{k}^{\prime} z_{k}^{\prime}}-L_{y_{k}^{\prime}}, \\
& \left(-i \hbar \frac{\partial}{\partial \Phi}\right)_{x_{k} y_{k}^{z} z_{k}}=\left(-i \hbar \frac{\partial}{\partial \Phi}\right)_{x_{k}^{\prime} y_{k}^{\prime} z_{k}^{\prime}}+\sin \Theta L_{x_{k}^{\prime}}-\cos \Theta L_{z_{k}^{\prime}},
\end{aligned}
$$

where the $L$ 's are electronic angular momentum operators-for example,

$$
L_{y_{A}^{\prime}}=-i \hbar\left(z_{A}^{\prime} \frac{\partial}{\partial x_{A}^{\prime}}-x_{A}^{\prime} \frac{\partial}{\partial z_{A}^{\prime}}\right) .
$$

At this point, let us assume that the basis functions $\phi_{k}\left(\overrightarrow{\mathrm{r}}_{k} ;\langle\overrightarrow{\mathrm{R}}\rangle\right)$ were chosen such that when they are expressed in rotating coordinates, they depend only on $\langle R\rangle=|\langle\vec{R}\rangle|$, and not on $\theta$ or $\Phi$. This is the normal property of basis functions used for the description of molecules in Hund's cases $a$ or $c$ (Ref. 16); such functions are in fact originally defined in the rotating frame, with at most a parametric dependence on $\langle\vec{R}\rangle$, and only later are they transformed by (34) into the space-fixed frame. For such functions, writing

$$
\phi_{k}\left(\overrightarrow{\mathbf{r}}_{k} ;\langle\overrightarrow{\mathbf{R}}\rangle\right)=\phi_{k}^{\prime}\left(\overrightarrow{\mathbf{r}}_{k}^{\prime} ;\langle R\rangle\right),
$$

we have from Table III and Eqs. (35) and (36), 


$$
\begin{array}{r}
\Pi_{j k}^{R}\langle\langle R\rangle)=-i \hbar \int \phi_{j}^{\prime}\left(\overrightarrow{\mathrm{r}}_{j}^{\prime} ;\langle R\rangle\right)\left(\frac{\partial}{\partial\langle R\rangle} \phi_{k}^{\prime}\left(\overrightarrow{\mathrm{r}}_{k}^{\prime} ;\langle R\rangle\right)\right)_{\overrightarrow{\mathbf{r}}_{k}^{\prime}} d \overrightarrow{\mathrm{r}}_{j}^{\prime}, \\
\Pi_{j k}^{\Theta}\langle\langle R\rangle)=-\langle R\rangle^{-1} \int \phi_{j}^{\prime}\left(\overrightarrow{\mathbf{r}}_{j}^{\prime} ;\langle R\rangle\right) L_{y_{k}^{\prime}} \phi_{k}^{\prime}\left(\overrightarrow{\mathrm{r}}_{k} ;\langle R\rangle\right) d \overrightarrow{\mathbf{r}}_{j}^{\prime}, \quad(39 \mathrm{~b}) \\
\Pi_{j k}^{\Phi}\langle\langle R\rangle)=\langle R\rangle^{-1}\left[\int \phi_{j}^{\prime}\left(\overrightarrow{\mathrm{r}}_{j}^{\prime} ;\langle R\rangle\right) L_{x_{k}^{\prime}} \phi_{k}^{\prime}\left(\overrightarrow{\mathrm{r}}_{k}^{\prime} ;\langle R\rangle\right) d \overrightarrow{\mathbf{r}}_{j}^{\prime}\right. \\
\left.-\cot \int \phi_{j}^{\prime}\left(\overrightarrow{\mathrm{r}}_{j j}^{\prime} ;\langle R\rangle\right) L_{z_{k}^{\prime}} \phi_{k}^{\prime}\left(\overrightarrow{\mathrm{r}}_{k}^{\prime} ;\langle R\rangle\right) d \overrightarrow{\mathbf{r}}_{j}^{\prime}\right] .
\end{array}
$$

For any such rotating basis functions, all coupling matrices are functions only of $\langle R\rangle$ [except for $\Pi^{\Phi}$, which has the $\cot \Theta$ dependence indicated in $(39 \bar{c})]$.

\section{The radial component-corrected nonadiabatic couplings}

The radial component, $\Pi^{R}$, differs substantially from the $P^{R}$ matrix that appears in the original formulation of PSS theory. In $\mathrm{Eq}$. (39a), we can again ignore the distinction between $\langle R\rangle$ and the internuclear separation $R$, because that distinction only involves corrections of order $(m / \mu)$. As was noted below Eqs. (12) and (23), the derivative $(\partial / \partial R)$ is to be evaluated holding $\overrightarrow{\mathbf{r}}_{k}^{\prime}$ fixed; hence it represents the rate of change of $\phi_{k}^{\prime}$ as seen in a frame that is moving with the center to which $\phi_{k}^{\prime}$ is attached. Such change of $\phi_{k}^{\prime}$ comes only from the parameters, like orbital exponents, that are permitted to vary with internuclear separation to optimize the basis. If such parameters are all held fixed, then $\Pi^{R}$ vanishes exactly at all internuclear separations.

This may be contrasted with the behavior of the $P^{R}$ matrix; in Eq. (2b) we see that it is defined similarly to (39), but the derivative is evaluated holding $\overrightarrow{\mathbf{r}}^{\prime}$ fixed; hence it involves the rate of change of $\phi_{k}$ as seen in a frame fixed on the center of mass of the nuclei. In that frame, the total change of $\phi_{k}^{\prime}$ arises not only from variation of optimized parameters, but also from the simple displacement of $\phi_{k}$ with its center. Such "displacement couplings" do not arise in the present formulation, and we call them "fictitious".

\section{The angular components-corrected rotational couplings}

In Eqs. (39b) and (39c), we see that the angular components involve operators representing electronic angular momenta about axes that pass through the center with which $\phi_{k}$ is associated. For example, one such matrix element is

$$
\int \phi^{*}\left(2 p_{z_{B}^{\prime}}\right) L_{y_{A}^{\prime}} \phi\left(2 p_{x_{A}^{\prime}}\right) d \overrightarrow{\mathbf{r}}
$$

with $L_{y_{A}^{\prime}}$ given in (37). The uncorrected PSS the- ory gives a similar matrix element of the operator $L_{y^{\prime}}$, where the $y^{\prime}$ axis passes through the center of mass of the nuclei.

The same corrected couplings have been known for a long time in the classical-trajectory formulation. For example, using the notation of Ref. 4, a class $F$ or class $V$ basis function must be multiplied by a corresponding electron-translation factor, which describes the momentum and kinetic energy of the electron as it is carried along with a given center. The action of the electronic Hamiltonian on this factor gives a term $\vec{A}_{F}$, and one can show that, [except for the neglected $(m / \mu)$ corrections]

$$
\underline{\vec{I}}=\underline{\overrightarrow{\mathbf{P}}}+\underline{\vec{A}}_{F} \text {. }
$$

This holds for both radial and angular components. (The quantum framework of Ref. 3 also gave the same correction to the angular components, but the correction to the radial component was rather more complicated, and only in the limit as $R \rightarrow \infty$ are those corrections identical to the ones obtained here.)

\section{Second-derivative matrix, $B$}

From Table III, or Eq. (23e) we see that the second-derivative matrix differs from the conventional one in the same way that $\vec{\Pi}$ differs from $\overrightarrow{\mathrm{P}}$; derivatives of $\phi_{k}$ are to be evaluated holding $\overrightarrow{\mathrm{r}}_{k}$ fixed. ${ }^{17}$ Hence, for example, if there are no $R$ dependent parameters in $\phi_{k}$, then the radial term, $\int \phi_{j}\left(\partial^{2} / \partial R^{2} \phi_{k}\right)_{\overrightarrow{\mathbf{r}}_{k}} d \overrightarrow{\mathbf{r}}_{j}$, vanishes exactly. Angular terms, however, can be quite complicated, because one must transform $\left(\nabla_{R}^{2}\right)_{\vec{r}_{k}}$ to $\left(\nabla_{R}^{2}\right)_{r_{k}^{\prime}}$ using (36). The result is messy, but well-known. ${ }^{1,18}$ Fortunately, one usually finds that these terms are unimportant.

The conventional $B$ matrix has often been calculated by the formula ${ }^{1}$

$$
-i \hbar \vec{\nabla} \cdot \overrightarrow{\mathbf{P}}+\underline{\overrightarrow{\mathbf{P}}} \cdot \underline{\overrightarrow{\mathbf{p}}},
$$

which is derived by putting $1=\sum_{n}\left|\phi_{n} \times \phi_{n}\right|$ between the two $\vec{\nabla}_{R}$ 's. No such relationship holds for the present $B$ matrix, however, because of the difference between $\left(\vec{\nabla}_{R}\right)_{\overrightarrow{\mathbf{r}}_{k}}$ and $\left(\vec{\nabla}_{R}\right)_{\overrightarrow{\mathbf{r}}_{j} \cdot}$ [Later, however, we will show how a formula related to (41) can be used in a special case.]

\section{Momentum-transfer coupling matrix, $\vec{\gamma}$}

The momentum-transfer coupling matrix $\vec{\gamma}$ de rives its name from the role it plays in the classical trajectory framework. Momentum-transfer factors (denoted $F_{j}^{*} F_{k}$ in Ref. 4) are responsible for the rapid decrease in charge-exchange cross sections at high velocities $\left(v_{\text {nuc }} \gg v_{e l}\right) .^{5}$ When these factors are expanded in a Taylor series, one finds 
that at low velocities they give a coupling matrix $\vec{\gamma}$ that is equivalent to the present one (except for the usual, unimportant $m / \mu$ corrections). This equivalence follows from an approximation derived from (19b),

$$
\overrightarrow{\mathrm{S}}_{k}=\kappa_{k} \overrightarrow{\mathrm{r}}-\frac{1}{2} \kappa_{k}^{2} \overrightarrow{\mathrm{R}}+\mathcal{O}(\mathrm{m} / \mu),
$$

with $\kappa_{k}=\frac{1}{2}\left(f_{k}+\lambda\right), f_{B}=1, f_{A}=-1$. This formula corresponds to Eq. (III 6) of Ref. 4. ${ }^{19}$ [Another useful formula follows from (42):

$$
\overrightarrow{\mathrm{s}}_{B}-\overrightarrow{\mathrm{s}}_{A}=\overrightarrow{\mathrm{r}}-\frac{1}{2} \lambda R \equiv \overrightarrow{\mathrm{r}}_{g} \text {, }
$$

which shows that $\vec{\sigma}$ is related to a dipole operator, but one which only connects states on different centers.]

Intuitively, one expects momentum-transfer effects to be small at low collision velocities. Nevertheless, like $\vec{\Pi}, \vec{\gamma}$ is in principle of order $\hbar / a_{0}$, so the re is no proof that it is always negligible.

\section{CHANGE OF REPRESENTATION}

We have now derived the second-order coupled equations (29) that arise from the ansatz (6), and we have briefly examined each of the coupling matrices that appear in the se equations. Any method of solution of these coupled equations can now be used to obtain the scattering matrix, and the associated transition probabilities and cross sections. A serious problem remains, however. For many real systems, an acceptable expansion of $\Psi$ in single-center states requires a large number of terms. Integration of such a large number of coupled equations is a task that may be prohibitive or hopeless.

Hence we must seek means by which the number of coupled equations can be reduced. Of course, we already know that many systems can be adequately described by a small set of coupled equations, provided that the basis functions are very carefully selected. For example, sharp truncation is frequently possible if the basis states are adiabatic (or, in some cases, partially diabatic). However, such states do not, in general, have single-center character, so they cannot be used directly in the ansatz (6).

There is a simple solution to this problem, and it requires only that we focus our attention on the final coupled equations (29), and not on the underlying theory from which they came. As soon as the various coupling matrices have been obtained in the original basis of single-center states, those matrices can be transformed to any other representation by certain rules. Let $\left\{\phi_{n}^{1}\right\}$ be our original set of single-center basis functions, and let $\left\{\phi_{\mu}^{2}\right\}$ be obtained from $\left\{\phi_{n}^{1}\right\}$ by arbitrary, and perhaps $R$-dependent, invertible transformations:

$$
\phi_{\mu}^{2}=\sum_{n} U_{n \mu} \phi_{n}^{1}
$$

Then the form and the substance of the coupled equations (29) is invariant under transformations defined by

$$
\begin{aligned}
& \underline{\chi}^{2}=\underline{U}^{-1} \underline{\chi}^{1}, \\
& M^{2}=\underline{U}^{+} \underline{M}^{1} U,
\end{aligned}
$$

for all matrices $\underline{M}$ except

$$
\begin{aligned}
& \vec{\Pi}^{2}=U^{+} \vec{\Pi}^{1} U-i \hbar \underline{U} \underline{S}^{1} \vec{\nabla}_{R} U, \\
& \underline{B}^{2}=\underline{U}^{+} \underline{B}^{1} \underline{U}-2 i \hbar \underline{U^{+}}\left(\underline{\Pi}^{1}+\vec{\gamma}^{1}\right) \cdot \vec{\nabla} U-\hbar^{2} \underline{U}^{+} \underline{S}^{1} \nabla^{2} U .
\end{aligned}
$$

Whenever we can find linear transform ations such that only a few equations are strongly coupled in the new representation, then the problem is solved. In most cases, the transformation is taken to diagonalize $\underline{h}$, or all but a small part of $h$.

Looking at the same problem from the opposite point of view, suppose we have obtained eigenfunctions of $h$, or suppose we have found some other "good" set of electronic functions $\phi_{\mu}^{2}$ such that an adequate expansion of $\Psi$ requires only a few terms. Then to solve the collision problem, we require displacement-corrected coupling matrices $\underline{\Pi}, \underline{B}$, and $\vec{\gamma}$. One way to obtain them is to decompose the functions $\phi_{\mu}^{2}$ into single-center states as in (44), evaluate $\underline{\Pi}^{1}, \underline{\gamma}^{1}$, and $\underline{B}^{1}$ as discussed earlier, and then transform back using Eqs. (45). ${ }^{20}$ In most cases, in fact, the functions $\phi_{\mu}^{2}$ would have been derived by a variational calculation involving expansion in single-center states, so in principle the required decomposition would already be available. This is the way the present theory would normally be used in a computation.

Two special results should be mentioned. If the eigenfunctions are given in terms of a "class $F$ " basis, in which the elementary basis functions have no parametric dependence on $R$, then the radial part of the nonadiabatic coupling matrix arises only from the $R$ dependence of the coefficients, ${ }^{21}$

$$
\underline{\Pi}^{2, R}=-i \hbar \underline{U}^{+} \underline{S}^{1} \frac{d \underline{U}}{d R} .
$$

In the same case, if the term $-2 i \hbar U^{+} \vec{\gamma}^{1} \cdot \vec{\nabla}_{R} U$ can be neglected, then the radial part of the secondderivative matrix, $B$, also arises only from the coefficients, and it can be evaluated by a formula related to $(41)$,

$$
\begin{aligned}
-\hbar^{2} \underline{U}^{\dagger} \underline{S}^{1} d^{2} \underline{U} / d R^{2}= & \left.-i \hbar \frac{d}{d R} \underline{\Pi}^{2, R}+\underline{\Pi}^{2, R}+i \hbar d \underline{S}^{2} / d R\right) \\
& \times\left(\underline{S}^{2}\right)^{-1} \underline{\Pi}^{2, R} \simeq \underline{B}^{2, R},
\end{aligned}
$$


which is derived by differentiating (46a).

There is still another way to look at these changes of representation. Suppose that with each of the new basis functions $\phi_{\mu}^{(2)}$, we associate a curvilinear heavy-particle coordinate $\overrightarrow{\mathbf{R}}_{\mu}^{(2)}$ such that (in mass-scaled coordinates)

$$
\begin{aligned}
\left(\overrightarrow{\mathrm{S}}_{\mu}^{m}\right)^{(2)} \phi_{\mu}^{(2)} & \equiv\left[\left(\overrightarrow{\mathrm{R}}_{\mu}^{m}\right)^{(2)}-\overrightarrow{\mathrm{R}}^{m}\right] \phi_{\mu}^{(2)} \\
& =\sum_{n} U_{n \mu}\left(\overrightarrow{\mathrm{S}}_{n}^{m}\right)^{(1)} \phi_{n}^{(1)},
\end{aligned}
$$

where $\left(\overrightarrow{\mathrm{S}}_{n}^{m}\right)^{(1)}$ is the $\overrightarrow{\mathrm{S}}_{n}^{m}$ associated with the singlecenter basis function $\phi_{n}^{(1)}$. It then follows that, if we define

$$
\begin{aligned}
& \Psi^{(2)} \equiv \sum_{\mu}\left(\chi_{\mu}^{m}\right)^{(2)}\left[\left(\overrightarrow{\mathrm{R}}_{\mu}^{m}\right)^{(2)}\right] \phi_{\mu}^{(2)}\left[\overrightarrow{\mathrm{r}}_{\mu}^{m} ;\left(\overrightarrow{\mathrm{R}}_{\mu}^{m}\right)^{(2)}\right] \\
& \Psi^{(1)} \equiv \sum_{n}\left(\chi_{n}^{m}\right)^{(1)}\left[\left(\overrightarrow{\mathrm{R}}_{n}^{m}\right)^{(1)}\right] \phi_{n}^{(1)}\left[\overrightarrow{\mathrm{r}}_{n}^{m} ;\left(\overrightarrow{\mathrm{R}}_{n}^{m}\right)^{(1)}\right]
\end{aligned}
$$

then

$$
\Psi^{(2)}=\Psi^{(1)}+O(m / \mu)
$$

Equation (48a) is an ansatz having different curvilinear coordinates for different states. The general theory of such ansatzen is fundamentally the same as the theory presented in this paper, but certain details of the general theory are rather more complicated. Already, however, we see that (47) provides a link between the present framework and such a general theory.

\section{MULTIELECTRON SYSTEMS}

As was stated in the introduction, the theory developed here can also be applied to multielectron systems. Let us consider now how this may be done.

At the outset, we assumed that the elementary basis functions for the one-electron case must have single-center character. Similarly, for the many-electron case, we assume that the character of the basis functions is such that each electron is definitely associated with a particular center. Various electrons can be on different centers, and the multielectron basis function need not be a simple product of one-electron functions. Even $r_{i j}$-correlated basis functions are permitted, but it must be possible to identify a specific center with which the $i$ th electron is associated. (For this purpose, the electrons must be imagined to be distinguishable.)

Some examples should make this restriction clear. For a two-electron system, a state having both electrons on one atom might be described by (Jacobi) coordinates shown in Fig. $4 \mathrm{a}: \overrightarrow{\mathrm{r}}_{1 A}$ goes

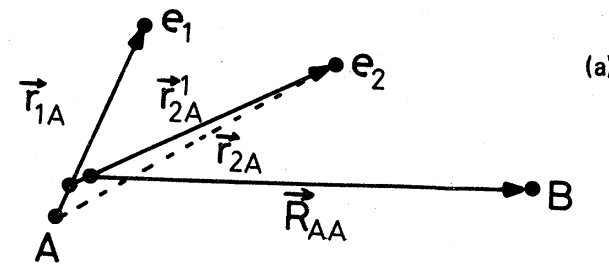

(a)

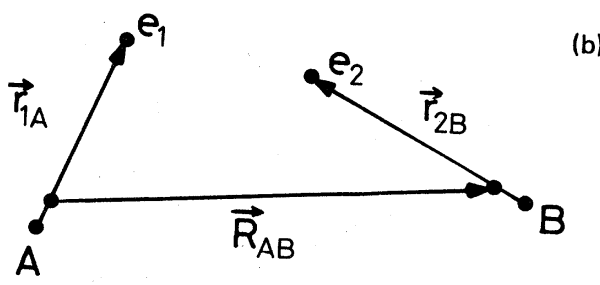

(b)

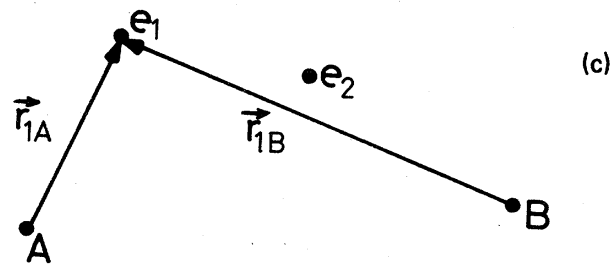

FIG. 4. Coordinates for a two-electron system。 (a) If both electrons are definitely associated with atom $A$, then the heavy particle coordinate $\overrightarrow{\mathbf{R}}_{A A}$ goes from the center of mass of $A$ to nucleus $B$. (b) If one electron is associated with each nucleus, then the heavy-particle coordinate connects the centers of mass of each atom. (c) If one electron is shared by both nuclei, as in an elliptical orbital, no unique Jacobi heavy-particle coordinate can be identified.

from nucleus $A$ to electron 1 , and $\vec{r}_{2 A}^{\prime}$ goes from their center of mass to electron 2. Any function of $\vec{r}_{1 A}$ and $\vec{r}_{2 A}^{\prime}$ has single-center $(A)$ character. (Hence we can also use the variables $\overrightarrow{\mathrm{r}}_{1 A}, \overrightarrow{\mathrm{r}}_{2 A}$, going from $A$ to each electron, at the price of introducing $\vec{\nabla}_{\vec{F}_{1 A}} \cdot \vec{\nabla}_{r_{2 A}}$ terms in $h_{k}$.) Also, any function $\phi\left(\overrightarrow{\mathrm{r}}_{1 A}, \overrightarrow{\mathrm{r}}_{2 B}\right)$ is also permitted, because electron 1 is definitely associated with $A$ and electron 2 with $B$. On the other hand, in an elliptical function like $\phi\left(\vec{r}_{1 A}, \vec{r}_{1 B}\right)$, electron 1 is not, in general, associated with a definite center, and the present methods may not be applicable.

If the basis functions have the character discussed above, then for each basis function $\phi_{k}$ the re is an unambiguous Jacobi heavy-particle coordinate $\vec{R}_{k}$ (Fig. 4). The ansatz (6) is generalized to

$$
\left.\Psi=\sum_{k=1}^{M} \chi_{k}\left(\overrightarrow{\mathrm{R}}_{k}\right) \phi_{k} \quad \text { (electron variables; } \overrightarrow{\mathrm{R}}_{k}\right)
$$


and, in the coordinates given for each term,

$$
H=-\frac{\hbar^{2}}{2 \mu_{k}} \nabla_{R_{k}}^{2}+h_{k} \text { 。 }
$$

That is all that is needed for carrying out the above development. So long as the "heavy-particle kinetic energy" appears as a separate term, as in (51), then all the rest of the equations (10) (29) follow, with only the obvious changes [ $S$ involves many-electron overlap integrals, $h_{k}$ is more complicated than $(9 \mathrm{~b}), L_{y k}$ becomes a sum of terms, one for each electron, etc.]

One aspect of this framework may be a little unusual: The elementary basis functions cannot, in general, be antisymmetrized, or they will lose the required character. However, after the basic matrices have been calculated in an unsymmetrized basis, antisymmetrization can be carried out as a special case of the linear transformation method discussed in Sec.VI. Hence, no fundamental problems arise from the antisymmetry requirement. Thus we see that multielectron systems introduce the expected challenging problems of implementation, but no new conceptual difficulties.

\section{CONCLUSION}

There is really only one fundamental postulate in this theory: It is that the ansatz (6) can provide a sufficiently accurate representation of $\Psi$ by means of a reasonable number of single-center functions. That ansatz leads directly to coupled integrodifferential equations (12), and we have shown that those integrodifferential equations can be reduced to coupled second-order differential equations (29). The only approximations involved in this reduction are (17), (24), and neglect of small terms in (28). All of these approximations involve retention of terms of order $\left(v_{\text {nuc }} / v_{\mathrm{e} 1}\right)$ $\simeq(\mathrm{m} / \mu)^{1 / 2}$ and neglect of some terms of order $\left(v_{\text {nuc }} / v_{\text {el }}\right)^{2} \simeq(m / \mu)$. These approximations should be applicable very generally for slow atomic collisions, in which condition $(20 a)$ or $(20 b)$ is satisfied.

Why should we believe that the ansatz (6) is a good one? More specifically, given any set of $M$ single-center functions $\left\{\phi_{k}\right\}$, that set can be used either in an ansatz of the form (1) or an ansatz of the form (6). Why should we think that of these two possibilities, (6) will normally give the better representation? There is no direct proof of the validity of this hypothesis, but there are several reasons for believing it to be correct. (i) As was already mentioned, by suitable choice of basis functions, individual terms in (6) can satisfy scattering boundary conditions exactly, while individual terms in (1) cannot. $^{3}$ (ii) The picture of inter- secting wave trains that arises from ansatz (6) seems intuitively much more reasonable than the picture that would arise fron ansatz (1) (see Fig. 2 and the discussion thereof.) (iii) We mentioned in the introduction that Eqs. (2) contain infinite-range couplings and other "fictitious" terms. The fact that such defects do not appear in Eqs. (12) or (29) again suggests that (6) is a better approximation than (1).

(iv) Finally, though our presentation of the theory involved only a quantum-mechanical framework, the most compelling arguments favoring ansatz (6) arise by examining the classical limit. In the classical-trajectory framework, it is assumed that the nuclei move along a classical path, and the electronic wave function $\Upsilon$ satisfies

$$
h(\overrightarrow{\mathrm{r}}, \overrightarrow{\mathrm{R}}(t)) \Upsilon=i \hbar \frac{\partial \Upsilon}{\partial t} .
$$

One way to solve this equation involves expansion such as

$$
\Upsilon=\sum_{k} d_{k}(t) \phi_{k}(\overrightarrow{\mathrm{r}} ; \overrightarrow{\mathrm{R}}(t))
$$

However, it is now well-established that a better expansion is obtained by including electron-translation factors, $F_{k}$ :

$$
\Upsilon=\sum_{k} d_{k}(t) F_{k} \phi_{k}(\overrightarrow{\mathrm{r}} ; \overrightarrow{\mathrm{R}}(t)),
$$

where (for single-center $\phi_{k}$ ) $F_{k}$ is typically of the form

$$
F_{k}=\exp \left[i m\left(\overrightarrow{\mathrm{v}}_{k} \cdot \overrightarrow{\mathbf{r}}-\frac{1}{2} v_{k}^{2} t\right)\right],
$$

and $\vec{v}_{k}$ is the velocity of the center with which $\phi_{k}$ is moving. Now, it was previously known that ansatz (1) corresponds in the classical limit to ansatz (53). One of the fundamental contributions of this paper is the recognition that the new ansatz (6) is the quantum analog of ansatz (54): The same displacement-corrections and momentum-transfer effects that arise from electron-translation factors in the classical-trajectory framework are obtained in a quantum framework by modifying the heavy-particle coordinate (see also Appendix A). It follows that all of the formal and computational evidence that favors (54) over (53) also favors (6) over (1).

The development given herein (and in Ref. 3) leads to a new conception of the definition and meaning of nonadiabatic couplings. ${ }^{22}$ In the earlier theoretical framework, of which Eqs. (1) and (2) were the foundation, nonadiabatic couplings were defined by the (usually Hermitian) matrix $\underline{\vec{P}}$, which 
represents the total rate of change of basis functions with internuclear distance. In the present formulation $\overrightarrow{\mathbf{P}}$ is replaced by $\vec{\Pi}$, and a new matrix, $\vec{\gamma}$, also appears. We may say that the (generally non-Hermitian) matrix II represents nonadiabatic couplings, and that $\vec{\gamma}$ represents momentum-transfer effects. $\vec{I}$ is the part of the total rate of change of the basis functions that represents distortion, polarization, or change of character: In a singlecenter basis, $\overrightarrow{I I}$ arises only from coefficients or nonlinear parameters that vary with $R$. Equivalently, $\vec{I}$ involves the rate of change of a basis function as seen in a frame of reference that is moving with the center to which that basis function is attached. Or, yet again, $\vec{\Pi}$ represents the total rate of change of the basis functions [as seen in the CMN (center of mass of the nuclei) frame] minus the part of the total rate of change that only corresponds to displacement of the orbitals with the moving nuclei.

Now, given a general molecular basis function $\phi(\overrightarrow{\mathrm{r}} ; \overrightarrow{\mathrm{R}})$ and its rate of change, $\vec{\nabla}_{R} \phi(\overrightarrow{\mathrm{r}} ; \overrightarrow{\mathrm{R}})$, it is not always possible to identify uniquely the part of $\vec{\nabla}_{R} \phi$ that represents displacement. (For example, displacement effects have sometimes been identified by using switching functions, for which various definitions are possible.) It follows that nonadiabatic couplings are also, in principle, nonunique. However, the displacement properties of singlecenter functions are, by definition, clear and unambiguous. It follows that a representation of $\Psi$ in single-center functions permits definite identification and elimination of displacement effects, and unambiguous evaluation of nonadiabatic and momentum-transfer couplings.

\section{ACKNOWLEDGMENTS}

This work has been supported by grants from the National Science Foundation (United States) and from the Nederlandse Organisatie voor ZuiverWetenschappelijk Onderzoek (Netherlands). The hospitality of the FOM Laboratory is gratefully acknowledged.

\section{APPENDIX A: THE CLASSICAL LIMIT}

We show here that when the present quantummechanical theory is taken to the classical limit, it leads to forms of the classical trajectory equations that incorporate corrections for displacement and momentum transfer. The simplest technique suitable for this purpose is to take the limit of large nuclear mass at fixed nuclear velocity. ${ }^{23}$ This method leads to the impact parameter form of the classical-trajectory equations.

\section{A. Uncorrected PSS theory}

The classical limit of the original form of PSS theory is well known. We set

$$
\chi(\vec{R})=\exp (i \mu \overrightarrow{\mathrm{v}} \cdot \overrightarrow{\mathrm{R}} / \hbar) \underline{d}(\overrightarrow{\mathrm{R}}),
$$

where $\overrightarrow{\mathrm{v}}$ is a constant velocity in the $+Z$ direction with magnitude $|\overrightarrow{\mathrm{v}}|=(2 E / \mu)^{1 / 2}$. Putting this into (2), and sorting in powers of $\mu$, one immediately obtains

$$
[-i \hbar \underline{S} \overrightarrow{\mathrm{v}} \cdot \vec{\nabla}+(\underline{h}+\overrightarrow{\vec{v}} \cdot \underline{\vec{P}})] \underline{d}=O\left(\mu^{-1}\right)
$$

and setting the right-hand side to zero, we obtain an older form of the classical-trajectory equations. In this form, corrections for displacement and momentum transfer are not incorporated.

B. New coupled equations (29)

When the same analysis is performed on Eqs. (29), the result is immediately

$$
i \hbar \underline{\hbar} \underline{\vec{v}} \cdot \vec{\nabla} \underline{d}=[\underline{h}+\overrightarrow{\vec{v}} \cdot(\underline{\vec{\Pi}}+\underline{\vec{\gamma}})] \underline{d} .
$$

These are seen to be the same as Eqs. (III12) of Ref. 4 , since $\vec{I}=\overrightarrow{\mathbb{P}}+\vec{A}$ (and $\underline{\vec{\gamma}}$ here is essentially the same ${ }^{19}$ as $\overline{\bar{\gamma}}$ in Ref. 4.) In these equations, displacement and momentum-transfer effects are included to first order in $v$.

\section{Integrodifferential equations (12)}

What happens if the coupled integrodifferential equations (12) are taken to the classical limit by the same procedure? To answer this, let us simplify by assuming that the basis set $\left\{\phi_{k}\right\}$ contains only two types of functions: One set centered on nucleus $A,\left\{\phi_{k_{A}}\right\}$, and another set centered on nucleus $B,\left\{\phi_{k_{B}}\right\}$. The "coefficients" are similarly divided into $\left\{\chi_{k_{A}}\left(\overrightarrow{\mathrm{R}}_{A}\right)\right\}$ and $\left\{\chi_{k_{B}}\left(\overrightarrow{\mathrm{R}}_{B}\right)\right\}$. Now we set

$$
\begin{aligned}
& \chi_{k_{A}}=\exp \left(i \mu_{A} \overrightarrow{\mathrm{v}}_{A} \cdot \overrightarrow{\mathrm{R}}_{A} / \hbar\right) d_{k_{A}}\left(\overrightarrow{\mathrm{R}}_{A}\right), \\
& \chi_{k_{B}}=\exp \left(i \mu_{B} \overrightarrow{\mathrm{v}}_{B} \cdot \overrightarrow{\mathrm{R}}_{B} / \hbar\right) d_{k_{B}}\left(\overrightarrow{\mathrm{R}}_{B}\right) .
\end{aligned}
$$

The velocities $v_{A}$ and $v_{B}$ are velocities of relative motion along the heavy-particle coordinates $\overrightarrow{\mathrm{R}}_{A}$ and $\overrightarrow{\mathrm{R}}_{B}$; let us take

$$
\mu_{A} v_{A}^{2} / 2=\mu_{B} v_{B}^{2} / 2=\mu v^{2} / 2=E
$$

Now, of the set of equations (12), consider the one involving $\phi_{j_{B}}^{*}$ : 


$$
\begin{aligned}
& \int \phi_{j_{B}}^{*}\left(\overrightarrow{\mathrm{r}}_{B} ; \overrightarrow{\mathrm{R}}_{B}\right) \mathcal{O}(B)\left(\overrightarrow{\mathrm{r}}_{B}, \overrightarrow{\mathrm{R}}_{B}\right) d \overrightarrow{\mathrm{r}}_{B} \\
& =\int d \overrightarrow{\mathrm{r}}_{B} \phi_{J_{B}}^{*} \exp \left(i \mu_{A} \overrightarrow{\mathrm{v}}_{A} \cdot \overrightarrow{\mathrm{R}}_{A} / \hbar\right) \\
& \quad \times \sum_{k_{A}}\left\{\left(2 \mu_{A}\right)^{-1}\left[\phi_{k_{A}}\left(\mu_{A} \overrightarrow{\mathrm{v}}_{A}-i \hbar \vec{\nabla}_{R_{A}}\right)^{2}+2\left(-i \hbar \vec{\nabla}_{R_{A}} \phi_{k_{A}}\right) \cdot\left(\mu_{A} \overrightarrow{\mathrm{v}}_{A}-i \hbar \vec{\nabla}_{R_{A}}\right)-\hbar^{2} \nabla_{R_{A}}^{2} \phi_{k_{A}}\right]-\hbar^{2}\left(h_{A}-E\right) \phi_{k_{A}}\right\} d_{k_{A}} \\
& +\int d \overrightarrow{\mathrm{r}}_{B} \phi_{j_{B}}^{*} \exp \left(i \mu_{B} \overrightarrow{\mathrm{v}}_{B} \cdot \overrightarrow{\mathrm{R}}_{B} / \hbar\right) \\
& \quad \times \sum_{k_{B}}\left\{\left(2 \mu_{B}\right)^{-1}\left[\phi_{k_{B}}\left(\mu_{B} \overrightarrow{\mathrm{v}}_{B}-i \hbar \vec{\nabla}_{R_{B}}\right)^{2}+2\left(i \hbar \vec{\nabla}_{R_{B}} \phi_{k_{B}}\right) \cdot\left(\mu_{B} \overrightarrow{\mathrm{v}}_{B}-i \hbar \vec{\nabla}_{R_{B}}\right)-\hbar^{2} \nabla_{R_{B}}^{2} \phi_{k_{B}}\right]\right. \\
& \left.\quad+\left(h_{B}-E\right) \phi_{k_{B}}\right\} d_{k_{B}}=0 .
\end{aligned}
$$

Again sorting in powers of $\mu_{A}$ or $\mu_{B}$, the term containing $\mu_{A}$ or $\mu_{B}$ to the zeroth power is

$$
\begin{aligned}
\int d \overrightarrow{\mathrm{r}}_{B} \phi_{j_{B}}^{*} & \left(\sum_{k_{B}}\left[\phi_{k_{B}}\left(-i \hbar \overrightarrow{\mathrm{v}}_{B} \cdot \vec{\nabla}_{R_{B}}\right) d_{k_{B}}+d_{k_{B}}\left(h_{B}-i \hbar \overrightarrow{\mathrm{v}}_{B} \cdot \vec{\nabla}_{R_{B}}\right) \phi_{k_{B}}\right]\right. \\
& \left.+\exp \left[i\left(\mu_{A} \overrightarrow{\mathrm{v}}_{A} \cdot \overrightarrow{\mathrm{R}}_{A}-\mu_{B} \overrightarrow{\mathrm{v}}_{B} \cdot \overrightarrow{\mathrm{R}}_{B}\right) / \hbar\right] \sum_{k_{A}}\left[\phi_{k_{A}}\left(-i \hbar \overrightarrow{\mathrm{v}}_{A} \cdot \vec{\nabla}_{R_{A}}\right) d_{k_{A}}+d_{k_{A}}\left(h_{A}-i \hbar \overrightarrow{\mathrm{v}}_{A} \cdot \vec{\nabla}_{R_{A}}\right) \phi_{k_{A}}\right]\right)=0 .
\end{aligned}
$$

The exponential quantity can be evaluated using

$$
\overrightarrow{\mathrm{R}}_{A}=\left(\frac{M_{A}}{m_{0}+M_{A}}\right) \overrightarrow{\mathrm{R}}_{B}-\left(\frac{m_{0}}{m_{0}+M_{A}}+\frac{m_{0}}{m_{0}+M_{B}}\right) \overrightarrow{\mathrm{r}}_{B}
$$

and, using also (A5), it turns out to be

$$
\exp \left[-i m_{0} v \cdot\left(\overrightarrow{\mathrm{r}}_{B}+\frac{1}{2} \overrightarrow{\mathrm{R}}_{B}\right)\right] \text {. }
$$

From this point on, we can neglect the differences between $\vec{v}_{A}, \vec{v}_{B}$, and $\vec{v}$, and those between $\vec{R}_{A}, \vec{R}_{B}$, and $\vec{R}$, because those differences are of order $(m / \mu)$. It then follows that Eqs. (A7) become

$$
\underline{\vec{S}}(v)(-i \hbar \overrightarrow{\mathrm{v}} \cdot \vec{\nabla}) \underline{d}+[\underline{h}(v)+\overrightarrow{\mathrm{v}} \cdot \underline{\vec{I}}(v)] \underline{d}=0,
$$

where the "direct" matrix elements are defined as before,

$$
S_{j_{B} k_{B}}(v)=S_{j_{B} k_{B}}=\int \phi_{j_{B}}^{*} \phi_{k_{B}} d \overrightarrow{\mathrm{r}},
$$

etc., but "exchange" matrix elements contain the "momentum-transfer factor,"

$$
\begin{gathered}
S_{j_{B^{k}} A}(v)=\int \phi_{j_{B}}^{*} \exp \left(-i m \overrightarrow{\mathrm{v}} \cdot \overrightarrow{\mathrm{r}}_{g}\right) \phi_{k_{A}} d \overrightarrow{\mathrm{r}}, \\
h_{j_{B} k_{A}}(v)=\int \phi_{j_{B}}^{*} \exp \left(-i m \overrightarrow{\mathrm{v}} \cdot \overrightarrow{\mathrm{r}}_{g}\right) h \phi_{k_{A}} d \overrightarrow{\mathrm{r}}, \\
\vec{\Pi}_{j_{B^{k_{A}}}}(v)=\int \phi_{j_{B}}^{*} \exp \left(-i m \overrightarrow{\mathrm{v}} \cdot \overrightarrow{\mathrm{r}}_{g}\right) \\
\times\left(-i \hbar \vec{\nabla}_{R} \phi_{k_{A}}\right)_{r_{A}} d \overrightarrow{\mathrm{r}} .
\end{gathered}
$$

These equations are equivalent to Eqs. (III 4) of Ref. $4[\underline{\underline{I}}(v)=\underline{\vec{P}}(v)+\underline{\vec{A}}(v)]$. These are the exact classical-trajectory equations (including electrontranslation-factors) for single-center states and rectilinear trajectories.

\section{APPENDIX B: REMARKS ON A DIFFERENT} FORMULATION

Recently another approach to the theory of charge-exchange processes has been developed. ${ }^{24}$ That approach is similar to the present one insofar as it also makes use of single-center basis functions and coordinate transformations. The authors state that their proposed method "... resolves the formal and practical difficulties ... which have attended theories of charge exchange ..." and that it provides "... an exact and practical formulation of the proper close-coupled (truncated) solution." The present author regrets to report that he finds these claims to be too strong. He holds the view that the formulation given in Ref. 24 leaves certain fundamental difficulties unresolved, and that, as a result, the truncated equations given there are quite inappropriate for describing some processes. Some insight can be gained by examining the differences between that formulation and the present one.

(1) The ansatz for $\Psi$, Eq. (2.14) and (2.15) of Ref. 24a [or Eq. (10) of Ref. 24b] is a special case of our Eq. (1). In the present notation, that ansatz is

$$
\Psi=\sum_{k_{A}} \chi_{k_{A}}(\overrightarrow{\mathrm{R}}) \phi_{k_{A}}\left(\overrightarrow{\mathrm{r}}_{A}\right)+\sum_{k_{B}} \chi_{k_{B}}(\overrightarrow{\mathrm{R}}) \phi_{k_{B}}\left(\overrightarrow{\mathrm{r}}_{B}\right),
$$

with $\phi_{k_{A}}$ and $\phi_{k_{B}}$ being (nonorthogonal) atomic- $A$ and $-B$ states. The important thing is that the "coefficients" $\left(\chi_{k_{A}}, \chi_{k_{B}}\right)$ are defined to be functions of $\vec{R}$, not of $\vec{R}_{A}$ and $\vec{R}_{B}$ respectively. Accordingly, Eq. (B1) is a generalization of our Eq. (8b), and it implies the same kind of picture. The basic pos- 
tulate of the present approach is that

$$
\Psi=\sum_{k_{A}} \chi_{k_{A}}\left(\overrightarrow{\mathrm{R}}_{A}\right) \phi_{k_{A}}\left(\overrightarrow{\mathrm{r}}_{A}\right)+\sum_{k_{B}} \chi_{k_{B}}\left(\overrightarrow{\mathrm{R}}_{B}\right) \phi_{k_{B}}\left(\overrightarrow{\mathrm{r}}_{B}\right)
$$

is a better ansatz, and it is shown that the difficulties which appear in earlier theories of chargeexchange arise from the difference between Eqs. (B1) and (B2).

(2) It follows from Eq. (B1) that the coupled equations (2.18) ${ }^{24(a)}$ are a form of our Eqs. (2), except that the nonorthogonality of the basis is taken into account. For a nonorthogonal basis, Eqs. (2) become

$$
\left\{(2 \mu)^{-1}\left[\underline{S}(-i \hbar \nabla)^{2}+2 \underline{\overrightarrow{\mathrm{P}}} \cdot(-i \hbar \vec{\nabla})+\underline{B}\right]+\underline{h}-\underline{S} E\right\} \underline{\chi}=0 .
$$

By transforming from the scaled coordinates of Ref. 24(a) into unscaled coordinates, and by using the fact that

$$
\begin{aligned}
& {\left[\vec{\nabla}_{R}+\frac{1}{2}( \pm 1+\lambda) \vec{\nabla}_{r}\right] \phi_{k}=0,} \\
& +-B \text { state, } \\
& --A \text { state, }
\end{aligned}
$$

one finds that the matrix ${ }^{24(\mathrm{a})} \underline{\tilde{P}} \underline{C} / \mu$ or ${ }^{24(\mathrm{~b})} \otimes$ corre sponds to our $\underline{P}^{R} / \mu$ and the matrix ${ }^{24(a)} \underline{\tilde{Q}} \underline{C}^{\overline{2}} / \mu$ or $^{24(\mathrm{~b})} \underline{Q}$ corresponds to the radial term in our ma$\operatorname{trix} B / \mu$.

(3) Equation (2.18) $)^{24(a)}$ was said to be "an exact equation" for processes such as charge exchange. It is true that equations of that type follow rigorously from the given ansatz; however, the present author holds that those equations have deficiencies similar to those of the unmodified PSS theory. For example, the couplings due to $\underline{\tilde{P}} \underline{C}$ and $\underline{Q} \underline{C}^{2}$ are precisely the "fictitious (infinite-range) displacement couplings" that are eliminated in the present theory, and our "momentum-transfer term", $\underline{\vec{\gamma}}$, does not appear in (2.18). ${ }^{24(a)}$

It was suggested in Ref. 24 that the terms $\tilde{P} C$ and $\underline{Q} C^{2}$ may be ignored if the electron mas $\bar{s}$ is sufficiently smaller than the nuclear mass. This, however, only tends to obscure the difference between that formulation and the present one. First, it is not generally correct that ${ }^{24(b)}$ "both $\underline{Q}$ and $\rho$ represent corrections which are smaller by the ratio of the electron to the nuclear mass as compared to $H^{e}$." In the scheme presented here, since $\overrightarrow{\underline{P}}$ multiplies $\left(-i \hbar \nabla_{R}\right)$, the effect of this term is of $\operatorname{order}(m / \mu)^{1 / 2}$, not $(m / \mu)$. Second, although it is still true that there are many cases in which these terms are negligible, the present author holds that they basically should not appear in close-coupled equations. Real electronic transitions are not caused by these couplings; the terms reflect deficiencies of the ansatz, rather than the physics of the collision.
(4) We do not wish to imply by these remarks that there is some kind of error or mistake in Ref. 24. No such claim is made. As far as the present author can tell, those papers give correct analysis based on the ansatz (B1), so the methods of Ref. 24 must give accurate results whenever that ansatz is adequate. Furthermore, those papers correctly emphasize that much insight is gained by thinking of charge-exchange as a reactive-scattering problem.

The present author's claim is that (a) there are many cases (even for slow collisions) in which truncation of ansatz (B1) leads to significant inaccuracy, simply because the geometry of the ansatz is not well suited to the geometry of the potential, and (b) in those cases, ansatz (B2) should give better results.

(5) As one illustration of the difficulties that will be encountered in attempts to use the methods of Ref. 24 (or unmodified PSS methods in general), let us consider the case of charge exchange between protons and hydrogen atoms at relative energies of, say, $50 \mathrm{keV}$ :

$$
\mathrm{H}^{+}+\mathrm{H}(1 s) \rightarrow \mathrm{H}(1 s)+\mathrm{H}^{+} \text {. }
$$

This process has been quite accurately described ${ }^{5(b)}$ in the classical-trajectory framework using only a two-state basis $\left(\phi_{s_{A}}\right.$ and $\left.\phi_{s_{B}}\right)$. However, momentum-transfer factors were an essential part of the calculation, and it was shown that if they were neglected, the calculated exchange cross section would be much too large.

In Ref. 24, the matrix elements do not include momentum-transfer factors. Therefore, the effects of those factors must either be completely neglected, or at best, imitated by the effects of virtual transitions to excited states. In any case, this method cannot give an adequate description of process (B5) in this energy range by using only two basis states.

The present equations (29) also will not give a very accurate description of process (B5) in this energy range, but we can see the reason why; in these equations, momentum-transfer effects are included only to first order. On the other hand, the integrodifferential equations (12) do give a good description, for it was proved in Appendix A that they reduce to the exact classical-trajectory equations including momentum-transfer factors.

\section{APPENDIX C: FURTHER EXPLANATION OF THE STEP FROM (26a) TO (26b)}

For readers who do not find this step to be very obvious, we try to give a bit more explanation here. ${ }^{25}$ First, note that in this paper, we are consistently using "mathematician's notation" for changes of variable: if 


$$
\chi_{1}^{m}\left(\overrightarrow{\mathbf{R}}_{\mathbf{1}}^{m}\right)=\exp \left(i \overrightarrow{\mathrm{k}} \cdot \overrightarrow{\mathbf{R}}_{\mathbf{1}}^{m}\right)
$$

then

$$
\chi_{1}^{m}\left(\overrightarrow{\mathbf{R}}_{2}^{m}\right)=\exp \left(\overrightarrow{i \overrightarrow{\mathrm{k}}} \cdot \overrightarrow{\mathrm{R}}_{2}^{m}\right)
$$

regardless of the relationship between $\vec{R}_{1}^{m}$ and $\vec{R}_{2}^{m}$. Now suppose ansatz (6) contains just two terms

$$
\Psi=\chi_{A}\left(\overrightarrow{\mathrm{R}}_{A}^{m}\right) \phi_{A}+\chi_{B}\left(\overrightarrow{\mathrm{R}}_{B}^{m}\right) \phi_{B} .
$$

Then (26a) is two equations

$$
\begin{aligned}
& M_{A A}\left(\overrightarrow{\mathrm{R}}_{A}^{m}\right) \chi_{A}^{m}\left(\overrightarrow{\mathrm{R}}_{A}^{m}\right)+M_{A B}\left(\overrightarrow{\mathrm{R}}_{A}^{m}\right) \chi_{B}^{m}\left(\overrightarrow{\mathrm{R}}_{A}^{m}\right)=0, \\
& M_{B A}\left(\overrightarrow{\mathrm{R}}_{B}^{m}\right) \chi_{B}^{m}\left(\overrightarrow{\mathrm{R}}_{B}^{m}\right)+M_{B B}\left(\overrightarrow{\mathrm{R}}_{B}^{m}\right) \chi_{B}^{m}\left(\overrightarrow{\mathrm{R}}_{B}^{m}\right)=0 .
\end{aligned}
$$

The independent variable in the first equation is $\overrightarrow{\mathrm{R}}_{A}^{m}$, and the equation holds for all values of $\overrightarrow{\mathrm{R}}_{A}^{m}$. Among other things, it holds when the components of $\overrightarrow{\mathrm{R}}_{A}^{m}$ are equal to $(0.98,2.317,1.64)$. The independent variable in the second equation is $\overrightarrow{\mathrm{R}}_{B}^{m}$, and the equation holds for all values of $\overrightarrow{\mathbf{R}}_{B}^{m}$. For example, it holds when the components of $\vec{R}_{B}^{m}$ are equal to $(0.98$, $2.317,1.64)$. Since both equations hold in general, they both hold if $\overrightarrow{\mathrm{R}}_{A}^{m}$ and $\overrightarrow{\mathrm{R}}_{B}^{m}$ should "accidentally" happen to have the same numerical value. But, for integration of those equations, it is most useful to compare surfaces in configuration space where $\overrightarrow{\mathrm{R}}_{A}^{m}$ and $\vec{R}_{B}^{m}$ do have the same numerical value. (The fact that they are distinct surfaces is not relevant to the integration.) Thus, if we let the numerical value of $\overrightarrow{\mathrm{R}}_{A}^{m}$ be denoted $\left\langle\overrightarrow{\mathrm{R}}^{m}\right\rangle$, then Eq. $\left(26 \mathrm{a}^{\prime}\right)$ is equivalent to

$$
M_{A A}\left\langle\overrightarrow{\mathbb{R}}^{m}\right\rangle \chi_{A}^{m}\left\langle\overrightarrow{\mathbb{R}}^{m}\right\rangle+M_{A B}^{m}\left\langle\overrightarrow{\mathbb{R}}^{m}\right\rangle \chi_{B}^{m}\left\langle\overrightarrow{\mathbb{R}}^{m}\right\rangle=0 .
$$

Then at the (distinct) configuration-space surface where $\vec{R}_{B}$ happens to be equal to $\langle\vec{R}\rangle$, Eq. $\left(26 a^{\prime \prime}\right)$ is equivalent to

$$
M_{B A}\left\langle\overrightarrow{\mathrm{R}}^{m}\right\rangle \chi_{A}^{m}\left\langle\overrightarrow{\mathrm{R}}^{m}\right\rangle+M_{B B}\left\langle\overrightarrow{\mathrm{R}}^{m}\right\rangle \chi_{B}^{m}\left\langle\overrightarrow{\mathrm{R}}^{m}\right\rangle=0 .
$$

Since Eqs. (26a') and (26a'l) must both be true, Eqs. $\left(26 b^{\prime}\right)$ and $\left(26 b^{\prime \prime}\right)$ must also be true. Furthermore, by constructing the solution to $\left(26 b^{\prime}\right)$ and $\left(26 b^{\prime \prime}\right)$, we have the solution to $\left(26 a^{\prime}\right)$ and $\left(26 a^{\prime \prime}\right)$. Therefore, (26a) and (26b) are equivalent.
*Permanent address: Physics Department, College of William and Mary, Williamsburg, Virginia.

${ }^{1}$ F. T. Smith, Phys. Rev. 179, 111 (1969).

${ }^{2}$ N. F. Mott and H. S. W. Massey, Theory of Atomic Collisions, 3rd ed. (Oxford University Press, London, 1965), p. 428.

${ }^{3}$ (a) W. R. Thorson and J. B. Delos, Phys. Rev. A 18 , 117 (1978); (b) 18, 135 (1978).

${ }^{4}$ J. B. Delos and W. R. Thorson, J. Chem. Phys. $\underline{70}$, 1774 (1979).

${ }^{5}$ (a) D. R. Bates and R. McCarroll, Proc. R. Soc. London Ser. A 245, 175 (1958); (b) R. McCarroll, Proc. R. Soc. London Ser. A 264, 547 (1961); (c) D. R. Bates and R. McCarroll, Adv. Phys. 11, 39 (1962).

${ }^{6}$ A. Riera and A. Salin, J. Phys. B 9, 2877 (1976).

${ }^{7}$ W. R. Thorson and H. Levy, II, Phys. Rev. 181, 232 (1969); H. Levy, II and W. R. Thorson, ibid. 181, 244 (1969); 181, 252 (1969); C. F. Lebeda, W. R. Thorson, and H. Levy, II, Phys. Rev. A 4 , 900 (1971); V. Sethu Raman, W. R. Thorson, and C. F. Lebeda, Phys. Rev. A 8,1316 (1973).

${ }^{8}$ M. E. Riley and T. A. Green, Phys. Rev. A 4, 619 (1971); T. A. Green, Phys. Rev. A 23, 519, 522 (1981); T. A. Green, E. J. Shipsay, and J. C. Browne, Phys. Rev. A 23, 546 (1981); also V. H. Ponce, J. Phys. B 12, 3731 (1979).

${ }^{9} \mathrm{~J}$. B. Delos, Revs. Mod. Phys. (in press).

${ }^{10}$ Reference 2, pp. 424-428. Note: Their equations were developed explicitly only for the special case corresponding to our Eq. (7), but our method is the same as theirs.

${ }^{11}$ Strictly speaking, in Ref. 4 , we defined a class $-F$ and class $-V$ basis as a set of single-center functions that rotate with the internuclear axis. In the present development, the states may rotate with the axes $\overrightarrow{\mathrm{R}}_{A}$ or $\overrightarrow{\mathrm{R}}_{B}$ (or they could be nonrotating).

${ }^{12}$ L. A. Pars, A Treatise on Analytical Dynamics (Heine- mann, London, 1965), p. 537.

${ }^{13}$ Even if the basis functions are rotating, they must be expressed as functions of space-fixed electronic coordinates $\left(\vec{r}_{A}\right.$ or $\left.\vec{r}_{B}\right)$, with the rotation described by the parametric dependence upon $\overrightarrow{\mathrm{R}}_{A}$ or $\overrightarrow{\mathrm{R}}_{B}$.

${ }^{14}$ We retain it because, although in the present context it is negligible, when changes of representation (Sec. VI) are permitted, one finds that there are some cases in which $\underline{B}$ can become locally large (curve crossings, for example).

${ }^{15}$ However, it does affect certain details of the form of the theory. If the whole theory were derived using unscaled coordinates, then the factor $\frac{1}{2}$ in the second term of $\vec{s}_{k}$ would not appear. However, one can show that if the approximations used in this theory are adequate, then that term affects only the absolute phases of the functions $\chi$, and not their magnitudes. Hence, transition probabilities would be unaffected by this change.

${ }^{16} \mathrm{G}$. Herzberg, Spectra of Diatomic Molecules, 2nd ed. (Van Nostrand, New York, 1950), p. 219 ff. In Hund's case $b$, the spatial part of the basis function rotates, but the spin part does not.

${ }^{17}$ To clearly distinguish between the two, we have denoted the present second-derivative matrix by a capital beta, while the conventional one is a capital $B$. This should be sufficient to eliminate all confusion.

${ }^{18}$ W. R. Thorson, J. Chem. Phys. 42, 3878 (1965); R. de L. Kronig, Band Spectra and Molecular Structure (Cambridge University Press, London, 1930), Chap. 1. ${ }^{19}$ Equation (III 6) of Ref. 4 appears to be different from Eq. (42) because in the former case the electronic coordinate was referred to an orig in at the geometric center of the molecule. If instead it had been referred to the CMN, then the two equations would be identical. ${ }^{20}$ This method is being studied by S. K. Knudson and W. R. Thorson. 
${ }^{21}$ In connection with Eq. (46a), we may note that the equation $\overrightarrow{\vec{P}}^{\dagger}-\overrightarrow{\vec{P}}=i \hbar \vec{\nabla} \underline{S}$ does not apply to $\vec{\pi}$. If the original basis is class $F$, we have instead, for the radial component $\left[\underline{\Pi}^{2}, R_{1}^{\dagger}\right]^{\dagger} \underline{\Pi}^{2, R}=i \hbar\left[d \underline{S}^{2} / d R-\underline{U}^{\dagger}\left(d \underline{S}^{1} / d R\right) \underline{U}\right]$.

${ }^{22}$ The concepts are "new" in the sense that they had not been developed in a fully quantum-mechanical framework before. However, the same corrections have been known for some time within the classical-trajectory formulation.

${ }^{23}$ M. E. Riley, Phys. Rev. A 7, 626 (1973).

${ }^{24}$ (a) E. B. Stechel, T. G. Schmalz, and J. C. Light, J. Chem. Phys. 70, 5640 (1979); (b) 70, 5660 (1979).
${ }^{25}$ The use of this step is not unprecedented. It was also taken by J. C. Y. Chen and K. M. Watson, Phys. Rev. $174,152(1968)$ in going from their equations (3.41) to (3.43). They expressed the argument very succinctly. Using our notation and equation numbers, their sentence is, "Equations $\left(26 \mathrm{a}^{\prime}\right)$ and $\left(26 \mathrm{a}^{\prime \prime}\right)$ may be written in more compact form if we simply write $\left\langle\overrightarrow{\mathrm{R}}^{m}\right\rangle$ for $\overrightarrow{\mathrm{R}}_{A}^{m}$ in the first, (and) $\left\langle\vec{R}^{m}\right\rangle$ for $\vec{R}_{B}^{m}$ in the second ..." (They used unscaled coordinates, and in other ways, their analysis is quite different from that given here, but our methods have this one step in common.) 